\title{
IMAGING SCHEMES FOR PERFECTLY CONDUCTING CRACKS *
}

\author{
HABIB AMMARI ${ }^{\dagger}$, JOSSELIN GARNIER ${ }^{\ddagger}$, HYONBAE KANG $^{\S}$, WON-KWANG PARK $₫$, \\ AND KNUT SØLNA\|
}

\begin{abstract}
We consider the problem of locating perfectly conducting cracks and estimating their geometric features from multi-static response matrix measurements at a single or multiple frequencies. A main objective is to design specific crack detection rules and to analyze their receiver operating characteristics and the associated signal-to-noise ratios. In this paper we introduce an analytic framework that uses asymptotic expansions which are uniform with respect to the wavelength-to-crack size ratio in combination with a hypothesis test based formulation to construct specific procedures for detection of perfectly conducting cracks. A central ingredient in our approach is the use of random matrix theory to characterize the signal space associated with the multi-static response matrix measurements. We present numerical experiments to illustrate some of our main findings.
\end{abstract}

Key words. asymptotic imaging, crack detection, imaging algorithms, random matrices, measurement noise, hypothesis testing, probability of detection

AMS subject classifications. 78M $35,78 \mathrm{~A} 46,15 \mathrm{~B} 52$

1. Introduction. The focus of this paper is on imaging small perfectly conducting cracks from measurements at single or multiple frequencies. Our imaging approach is based on asymptotic formulas for the signature of the crack which are uniform with respect to the wavelength-to-crack size ratio.

We assume that we have coincident transmitter and receiver arrays of $n$ elements. The multi-static response (MSR) matrix is the transmit-receive responses of this array. The problem we consider is to detect, localize, and image the crack from the MSR matrix measurements at single or multiple frequencies in the presence of measurement noise. We construct different imaging functionals for imaging the cracks from MSR measurements at a single or multiple frequencies. In particular, MUSIC (which stands for MUltiple SIgnal Classification) and Kirchhoff-type algorithms are investigated. Applying the techniques of statistical hypothesis testing we derive a strategy for ruling on the presence/absence of a crack based on the introduced imaging functionals. We revisit Berens' modelling that was introduced in [7] and derive, using our asymptotic formulas for the signature of the crack, appropriate probability of detection functions. The detailed statistical analysis carried out in this paper shows that the probability of false alarm is given in terms of a TracyWidom distribution, which is a bell-shape function somewhat different from the Gaussian distribution usually applied. Finally we perform numerical experiments using the proposed algorithms to test their performance and efficiency.

The paper presents a general framework for imaging perfectly conducting cracks. It proposes efficient imaging algorithms and establishes an approach for hypothesis testing. The paper extends several recent results, concepts, and methods for imaging cracks. Crack detection algorithms using electrostatic measurements have been derived in $[11,10,17]$. In [5] a continuous model was considered and an asymptotic

${ }^{*}$ This work was supported by the Agence Nationale de la Recherche, NRF20090085987, NSF grant DMS0709389, and the research program 2010 of Kookmin University in Korea.

${ }^{\dagger}$ Department of Mathematics and Applications, Ecole Normale Supérieure, 45 Rue d'Ulm, 75005 Paris, France (habib.ammari@ens.fr).

${ }^{\ddagger}$ Laboratoire de Probabilités et Modèles Aléatoires \& Laboratoire Jacques-Louis Lions, Université Paris VII, 2 Place Jussieu, 75251 Paris Cedex 5, France (garnier@math.jussieu.fr).

$\S$ Department of Mathematics, Inha University, Incheon 402-751, Korea (hbkang@inha.ac.kr).

『 Department of Mathematics, Kookmin University, 861-1, Jeongneung-dong, Seongbuk-gu, Seoul 136-702, Korea (parkwk@kookmin.ac.kr).

\|Department of Mathematics, University of California, Irvine, CA 92697 (ksolna@math.uci.edu). 
expansion of the boundary perturbations that are due to the presence of a small crack was derived. Moreover, a MUSIC-type approach for locating the crack from boundary measurements at a single frequency was designed. It was also shown that the location and the length of the crack can be estimated from the projection onto the noise space and the first significant singular value of the MSR matrix while the direction of the crack can only be estimated from the second singular vector. In this paper we integrate and extend these techniques by introducing an analytic framework that uses asymptotic expansions which are uniform with respect to the wavelength-to-crack size ratio in combination with a hypothesis test based formulation to construct specific procedures for detection and characterization of cracks. A central ingredient in our approach is the use of random matrix theory to characterize the signal space associated with the MSR measurements.

The paper is organized as follows. In Section 2 an asymptotic formalism for crack imaging is established. Imaging functionals to locate the cracks are introduced in Section 3. Then, we present the test for detection of the crack, that is to rule on whether it is present or not. This crucial test is based on analysis of the singular values of the MSR matrix and is derived in Section 4. An extension of Berens' modelling for crack detection is given in Section 5. In Section 6 we use the singular vectors to estimate the location given that there is a crack present. In the location estimation we introduce a location dependent threshold to the test whether a search point is associated with a crack or not. In Section 7, an optimization algorithm for reconstructing the crack orientation is presented. We illustrate with some numerical examples in Section 8. The paper ends with a discussion in Section 9. Some background on the probabilistic framework together with a proof of the uniform asymptotic expansion of the effect of a small crack are given in the appendices.

2. Asymptotic Modelling of Cracks. We shall consider the case where the crack is perfectly conducting and the space dimension is 2 . The crack $\Sigma_{c}$ is a segment characterized by its (half) length $l_{c} \in \mathbb{R}^{+}$, location $\boldsymbol{x}_{c} \in \mathbb{R}^{2}$, and orientation $\boldsymbol{t}_{c} \in \mathbb{S}^{1}$ :

$$
\Sigma_{c}=\left\{\boldsymbol{x}_{c}+x \boldsymbol{t}_{c},-l_{c} \leq x \leq l_{c}\right\} .
$$

We assume a transducer array with the transducers located at $\left(\boldsymbol{x}_{j}=\left(x_{1}^{(j)}, 0\right) ; j=\right.$ $1, \ldots, n)$, moreover, that the full MSR matrix is available. First, we assume that there is no noise. The governing equation for the time-harmonic field $u^{(j)}$ emitted by a source at $\boldsymbol{x}_{j}$ is

$$
\left\{\begin{array}{l}
\Delta u^{(j)}+\frac{\omega^{2}}{c_{0}^{2}} u^{(j)}=-\delta_{\boldsymbol{x}_{j}} \quad \text { in } \mathbb{R}^{2} \backslash \Sigma_{c} \\
u^{(j)}=0 \text { on } \Sigma_{c} \\
\left|\left(\frac{\partial}{\partial|\boldsymbol{x}|}-i \frac{\omega}{c_{0}}\right)\left(u^{(j)}(\boldsymbol{x})-\hat{G}\left(\omega, \boldsymbol{x}, \boldsymbol{x}_{j}\right)\right)\right|=\mathrm{O}\left(|\boldsymbol{x}|^{-3 / 2}\right)
\end{array}\right.
$$

where $\hat{G}$ is the time-harmonic Green's function

$$
\hat{G}(\omega, \boldsymbol{x}, \boldsymbol{y})=\frac{i}{4} H_{0}^{(1)}\left(\frac{\omega}{c_{0}}|\boldsymbol{y}-\boldsymbol{x}|\right),
$$

using the notation $|\boldsymbol{y}|=\|\boldsymbol{y}\|_{2}$. Here, $H_{0}^{(1)}$ is the zeroth order Hankel function of the first kind.

We assume a high-frequency regime in which the distance $L$ from the array center point to the crack is much larger than the wavelength, which is itself much larger than the crack length $l_{c}$ :

$$
\frac{\omega L}{c_{0}} \gg 1, \quad \varepsilon_{\omega}:=\frac{\omega l_{c}}{c_{0}} \ll 1 .
$$


We define the MSR matrix entries by $A_{j l}:=u^{(j)}\left(\boldsymbol{x}_{l}\right)-\hat{G}\left(\omega, \boldsymbol{x}_{l}, \boldsymbol{x}_{j}\right)$ for $j, l=1, \ldots, n$. In Appendix B we prove that the following asymptotic formula is valid when (2.3) holds:

$$
\begin{aligned}
A_{j l}(\omega)= & \frac{i c_{0}}{4 \omega \sqrt{\left|\boldsymbol{x}_{j}-\boldsymbol{x}_{c}\right|\left|\boldsymbol{x}_{l}-\boldsymbol{x}_{c}\right|}} \exp \left(\frac{i \omega}{c_{0}}\left(\left|\boldsymbol{x}_{j}-\boldsymbol{x}_{c}\right|+\left|\boldsymbol{x}_{l}-\boldsymbol{x}_{c}\right|\right)\right) \\
& \times\left[\frac{1}{\ln \left(\frac{\varepsilon_{\omega}}{4}\right)+\gamma-\frac{i \pi}{2}}-\frac{\varepsilon_{\omega}^{2}}{2} \frac{\left(\left(\boldsymbol{x}_{j}-\boldsymbol{x}_{c}\right) \cdot \boldsymbol{t}\right)\left(\left(\boldsymbol{x}_{l}-\boldsymbol{x}_{c}\right) \cdot \boldsymbol{t}\right)}{\left|\boldsymbol{x}_{j}-\boldsymbol{x}_{c}\right|\left|\boldsymbol{x}_{l}-\boldsymbol{x}_{c}\right|}+\mathrm{o}\left(\varepsilon_{\omega}^{2}\right)\right],
\end{aligned}
$$

where $\gamma \simeq 0.577$ is the Euler constant. Formula (2.4) gives not only the leadingorder term but also the second one in the perturbations due to the presence of the crack. It shows that the direction of the crack is a second-order information and therefore, harder to reconstruct than the location.

3. Estimation of Crack Location. We first recall some common imaging functionals, which are functions of a search point in the search domain where we are looking for cracks. In Section 6 we shall relate this to a location-dependent test that is derived in a probabilistic framework.

Note that in the presence of a crack at $\boldsymbol{x}_{c}$ the MSR matrix has the approximate form

$$
\mathbf{A} \simeq \tau\left(\omega, \boldsymbol{x}_{c}\right) \boldsymbol{d}\left(\omega, \boldsymbol{x}_{c}\right) \boldsymbol{d}\left(\omega, \boldsymbol{x}_{c}\right)^{T},
$$

with $\boldsymbol{d}\left(\omega, \boldsymbol{x}_{c}\right)$ the normalized illumination vector

$$
\boldsymbol{d}\left(\omega, \boldsymbol{x}_{c}\right)=\frac{1}{\sqrt{\sum_{j=1}^{n} \frac{1}{\left|\boldsymbol{x}_{j}-\boldsymbol{x}_{c}\right|}}}\left(\frac{1}{\sqrt{\left|\boldsymbol{x}_{j}-\boldsymbol{x}_{c}\right|}} \exp \left(\frac{i \omega}{c_{0}}\left|\boldsymbol{x}_{j}-\boldsymbol{x}_{c}\right|\right)\right)_{j=1, \ldots, n}
$$

and $\tau\left(\omega, \boldsymbol{x}_{c}\right)$ given by

$$
\tau\left(\omega, \boldsymbol{x}_{c}\right)=\frac{i c_{0} \sum_{j=1}^{n} \frac{1}{\left|\boldsymbol{x}_{j}-\boldsymbol{x}_{c}\right|}}{4 \omega\left(\ln \left(\frac{\varepsilon_{\omega}}{4}\right)+\gamma-\frac{i \pi}{2}\right)} .
$$

Here $T$ denotes the transpose. The first singular value and singular vectors of the Singular Value Decomposition (SVD) of the data

$$
\mathbf{A}=\mathbf{U} \boldsymbol{\Sigma} \overline{\mathbf{V}}^{T},
$$

satisfy $\mathbf{A} \boldsymbol{v}_{1}=\sigma_{1}^{(n)} \boldsymbol{u}_{1}$ with the relations

$$
\boldsymbol{u}_{1} \simeq \exp \left(i \theta_{1}\right) \boldsymbol{d}, \quad \boldsymbol{v}_{1} \simeq \exp \left(-i \theta_{2}\right) \overline{\boldsymbol{d}}, \quad \sigma_{1}^{(n)} \simeq\left|\tau\left(\omega, \boldsymbol{x}_{c}\right)\right|,
$$

with $\theta_{1}+\theta_{2}=\arg \left(\tau\left(\omega, \boldsymbol{x}_{c}\right)\right)$.

A MUSIC imaging functional at a single frequency $\omega$ is given by

$$
\mathcal{I}_{\mathrm{MU}}\left(\omega, \boldsymbol{x}^{S}\right):=\frac{1}{\left|\boldsymbol{d}\left(\omega, \boldsymbol{x}^{S}\right)-\left(\boldsymbol{u}_{1}(\omega), \boldsymbol{d}\left(\omega, \boldsymbol{x}^{S}\right)\right) \boldsymbol{u}_{1}(\omega)\right|},
$$

where $(\boldsymbol{a}, \boldsymbol{b})=\overline{\boldsymbol{a}} \cdot \boldsymbol{b}$. We contrast this with a more classical Kirchhoff migration formulation which migrates traces to the search point $\boldsymbol{x}^{S}$ :

$$
\mathcal{I}_{\mathrm{KM}}\left(\omega, \boldsymbol{x}^{S}\right):={\overline{\boldsymbol{d}\left(\omega, \boldsymbol{x}^{S}\right)}}^{T} \mathbf{A}(\omega) \overline{\boldsymbol{d}\left(\omega, \boldsymbol{x}^{S}\right)} .
$$

In terms of the SVD decomposition of the MSR matrix the Kirchhoff migration functional can be written as

$$
\mathcal{I}_{\mathrm{KM}}\left(\omega, \boldsymbol{x}^{S}\right)=\sum_{l=1}^{n}\left(\boldsymbol{d}\left(\omega, \boldsymbol{x}^{S}\right), \boldsymbol{u}_{l}(\omega)\right)\left(\boldsymbol{d}\left(\omega, \boldsymbol{x}^{S}\right), \overline{\boldsymbol{v}_{l}}(\omega)\right) \sigma_{l}^{(n)}(\omega) .
$$


In the case with one (sufficiently small) crack we have $\sigma_{1}^{(n)} \gg \sigma_{l}^{(n)}$, for all $l=$ $2, \ldots, n$, and the Kirchhoff migration functional is dominated by the contribution of the first singular value and vectors:

$$
\mathcal{I}_{\mathrm{KM}}\left(\omega, \boldsymbol{x}^{S}\right) \simeq\left(\boldsymbol{d}\left(\omega, \boldsymbol{x}^{S}\right), \boldsymbol{u}_{1}(\omega)\right)\left(\boldsymbol{d}\left(\omega, \boldsymbol{x}^{S}\right), \overline{\boldsymbol{v}_{1}}(\omega)\right) \sigma_{1}^{(n)}(\omega) .
$$

With measurements of the MSR matrix at multiple frequencies, $\left(\omega_{k}\right)_{k=1, \ldots, K}$, we can construct the imaging functional by summing over frequencies

$$
\mathcal{I}_{\mathrm{KMF}}\left(\boldsymbol{x}^{S}\right):=\frac{1}{K} \sum_{\omega_{k}}{\overline{\boldsymbol{d}\left(\omega_{k}, \boldsymbol{x}^{S}\right)}}^{T} \mathbf{A}\left(\omega_{k}\right) \overline{\boldsymbol{d}\left(\omega_{k}, \boldsymbol{x}^{S}\right)}
$$

where $K$ is the number of frequencies $\left(\omega_{k}\right)$. An alternative imaging functional when searching for a single crack is

$$
\mathcal{I}_{\mathrm{MT}}\left(\boldsymbol{x}^{S}\right):=\frac{1}{K} \sum_{\omega_{k}}\left(\boldsymbol{d}\left(\omega_{k}, \boldsymbol{x}^{S}\right), \boldsymbol{u}_{1}\left(\omega_{k}\right)\right)\left(\boldsymbol{d}\left(\omega_{k}, \boldsymbol{x}^{S}\right), \overline{\boldsymbol{v}_{1}}\left(\omega_{k}\right)\right)
$$

in which we have renormalized the information provided by the different modes, yet retained phase coherency. Finally, it is also possible to use a matched field imaging functional:

$$
\mathcal{I}_{\mathrm{MF}}\left(\boldsymbol{x}^{S}\right):=\frac{1}{K} \sum_{\omega_{k}}\left|{\overline{\boldsymbol{d}\left(\omega_{k}, \boldsymbol{x}^{S}\right)}}^{T} \mathbf{A}\left(\omega_{k}\right) \overline{\boldsymbol{d}\left(\omega_{k}, \boldsymbol{x}^{S}\right)}\right|^{2},
$$

in which the phase coherence between the different frequency-dependent components is not exploited. As we will see in Section 6, this makes sense when the different frequency-dependent components are incoherent.

4. Singular Values of the Symmetrized Noisy MSR matrix. The crack detection test that we will propose relies on the statistical properties of the MSR matrix in the presence of noise. Here we will make use of recent results of random matrix theory.

Consider the MSR matrix $\mathbf{A}$ in the presence of additive measurement noise:

$$
\mathbf{A}=\mathbf{A}_{0}+\mathbf{W}
$$

where $\mathbf{W}$ is a complex Gaussian matrix which models additive measurement noise. We assume that the entries of $\mathbf{W}$ are with mean zero and variance $a^{2}$. In the absence of a crack, the matrix $\mathbf{A}_{0}$ is $\mathbf{0}$. In the presence of a crack, the matrix $\mathbf{A}_{0}$ is assumed to be a rank-one matrix and we denote by $\sigma_{0}$ the nonzero singular value of $\mathbf{A}_{0}$. As shown in the previous section, this holds true if the crack is small and then $\sigma_{0}=\left|\tau\left(\omega, \boldsymbol{x}_{c}\right)\right|$.

We know by reciprocity that the response matrix in the absence of noise $\mathbf{A}_{0}$ is symmetric. In order to reduce the noise variance of the off-diagonal entries of $\mathbf{A}$, we consider the symmetrized matrix

$$
\mathbf{A}^{s}=\frac{1}{2}\left(\mathbf{A}+\mathbf{A}^{T}\right)=\mathbf{A}_{0}+\mathbf{W}^{s} .
$$

Then the entries of the symmetric matrix $\mathbf{W}^{s}$ are independent complex Gaussian random variables with mean zero and variance $a^{2} / 2$ off the diagonal $(j \neq l)$ and $a^{2}$ on the diagonal $j=l$. The symmetrization of the MSR matrix reduces the variance of its off-diagonal entries by a factor of $\sqrt{2}$. We introduce the parameter $\sigma_{\mathrm{c}}=\sqrt{n / 2} a$.

We denote by $\sigma_{1}^{(n)} \geq \sigma_{2}^{(n)} \geq \cdots \geq \sigma_{n}^{(n)}$ the singular values of the MSR matrix $\mathbf{A}^{s}$ sorted by decreasing order. We introduce the corresponding spectral measure:

$$
N^{(n)}\left(\left[\sigma_{u}, \sigma_{v}\right]\right)=\frac{1}{n} \operatorname{Card}\left\{j \in\{1, \ldots, n\}, \sigma_{j}^{(n)} \in\left[\sigma_{u}, \sigma_{v}\right]\right\} \text {, for any } \sigma_{u}<\sigma_{v} .
$$


Note that $N^{(n)}$ is a counting measure which consists of a sum of Dirac masses:

$$
N^{(n)}=\frac{1}{n} \sum_{j=1}^{n} \delta_{\sigma_{j}^{(n)}}
$$

The singular value distribution of the MSR matrix in the absence of crack. We first consider the symmetrized MSR matrix $\mathbf{A}^{s}$ in the absence of a crack.

Proposition 4.1. 1. When $n \rightarrow \infty$ the spectral measure $N^{(n)}$ almost surely converges to the absolutely continuous measure $N$ with compact support:

$$
N\left(\left[\sigma_{u}, \sigma_{v}\right]\right)=\int_{\sigma_{u}}^{\sigma_{v}} \rho(\sigma) d \sigma, \quad \rho(\sigma)=\frac{1}{\sigma_{\mathrm{c}}} \rho_{\mathrm{qc}}\left(\frac{\sigma}{\sigma_{\mathrm{c}}}\right),
$$

where $\rho_{\mathrm{qc}}$ is the quarter-circle law given by

$$
\rho(\sigma)=\frac{1}{\sigma_{\mathrm{c}}} \rho_{\mathrm{qc}}\left(\frac{\sigma}{\sigma_{\mathrm{c}}}\right), \quad \rho_{\mathrm{qc}}(\sigma)= \begin{cases}\frac{1}{\pi} \sqrt{4-\sigma^{2}} & \text { if } 0<\sigma \leq 2, \\ 0 & \text { otherwise. }\end{cases}
$$

2. When $n \rightarrow \infty$ the normalized $l^{2}$-norm of the singular values satisfies

$$
n\left[\frac{1}{n} \sum_{j=1}^{n}\left(\sigma_{j}^{(n)}\right)^{2}-\sigma_{\mathrm{c}}^{2}\right] \stackrel{\text { dist. }}{=} \mathcal{N}\left(\sigma_{\mathrm{c}}^{2}, 2 \sigma_{\mathrm{c}}^{4}\right),
$$

where $\mathcal{N}\left(\mu, \sigma^{2}\right)$ stands for the normal distribution with mean $\mu$ and variance $\sigma^{2}$, and $\stackrel{\text { dist. }}{=}$ means "equal in distribution".

Proof. The first point is standard in Random Matrix Theory [23]. The second point follows from the explicit representation of the $l^{2}$-norm of the singular values in terms of the entries of the matrix:

$$
\sum_{j=1}^{n}\left(\sigma_{j}^{(n)}\right)^{2}=\operatorname{Trace}\left({\overline{\left(\mathbf{A}^{s}\right)}}^{T} \mathbf{A}^{s}\right)=\sum_{j=1}^{n}\left|A_{j j}^{s}\right|^{2}+2 \sum_{j<l}\left|A_{j l}^{s}\right|^{2}
$$

and the use of the central limit theorem. Note that $\mathbb{E}\left(\left|A_{j j}^{s}\right|^{2}\right)=a^{2}, \operatorname{Var}\left(\left|A_{j j}^{s}\right|^{2}\right)=$ $a^{4}, \mathbb{E}\left(\left|A_{j l}^{s}\right|^{2}\right)=a^{2} / 2, \operatorname{Var}\left(\left|A_{j l}^{s}\right|^{2}\right)=a^{4} / 4$, for $j \neq l$, and $A_{j l}^{s}$ are the entries of the symmetric matrix $\mathbf{A}^{s}$. Here $\mathbb{E}$ stands for the expectation (mean value), Var for the variance.
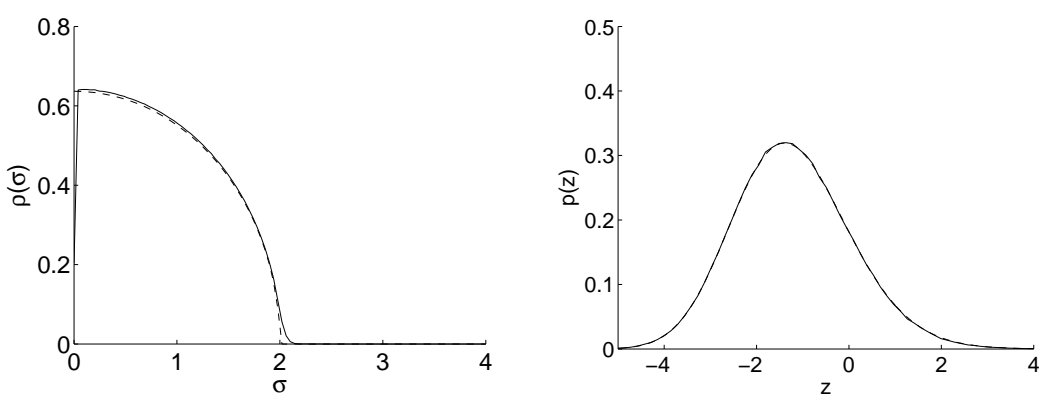

FIG. 4.1. Left figure: spectral measure for a complex symmetric Gaussian random matrix with $\sigma_{\mathrm{c}}=1$ obtained from Monte Carlo simulations with $n=50$ (solid) and compared with the theoretical quarter-circle law (dashed). Right figure: probability density function of the normalized maximal singular value $Z_{1}=2^{2 / 3} n^{2 / 3}\left(\max \sigma_{j}^{(n)} / \sigma_{\mathrm{c}}-2\right)$ obtained from Monte Carlo simulations with $n=50$ (solid) and compared with the theoretical Tracy-Widom distribution of type 1 (dashed). 
The largest singular value is $2 \sigma_{\mathrm{c}}$ up to a random correction of order $\sigma_{\mathrm{c}} n^{-2 / 3}$ :

$$
\sigma_{1}^{(n) \stackrel{\text { dist. }}{=}} \sigma_{\mathrm{c}}\left[2+2^{-2 / 3} n^{-2 / 3} Z_{1}+o\left(n^{-2 / 3}\right)\right]
$$

The random correction $Z_{1}$ follows a Tracy-Widom distribution of type 1 [20]:

$$
\begin{aligned}
& \mathbb{P}\left(Z_{1} \leq z\right)=\int_{-\infty}^{z} p_{\mathrm{TW} 1}(x) d x=\exp \left(-\frac{1}{2} \int_{z}^{\infty} \varphi(x)+(x-z) \varphi^{2}(x) d x\right), \\
& \mathbb{E}\left(Z_{1}\right) \simeq-1.21, \quad \operatorname{Var}\left(Z_{1}\right) \simeq 1.61,
\end{aligned}
$$

where $\varphi$ is the solution to the Painlevé equation

$$
\varphi^{\prime \prime}(x)=x \varphi(x)+2 \varphi(x)^{3}, \quad \varphi(x) \simeq \operatorname{Ai}(x), x \rightarrow \infty,
$$

Ai being the Airy function (see Fig. 4.1). Without the symmetrization of the MSR matrix we have a similar result but with a Tracy-Widom distribution of type 2 .

Note that the random correction to the maximal singular value has a relative amplitude of the order of $n^{-2 / 3}$, while the random correction to the $l^{2}$-norm has a relative amplitude of order $n^{-1}$. Let the ratio $R$ of the first singular value over the $l^{2}$-norm of the other singular values be defined by

$$
R:=\frac{\sigma_{1}^{(n)}}{\left(\frac{1}{n-1} \sum_{j=2}^{n}\left(\sigma_{j}^{(n)}\right)^{2}\right)^{1 / 2}} .
$$

Therefore, using Slutsky's theorem, we obtain the following result:

Proposition 4.2. In the absence of any crack, the ratio $R$ defined by (4.5) has the following statistical distribution

$$
R \stackrel{\text { dist. }}{=} 2+\frac{1}{2^{2 / 3} n^{2 / 3}} Z_{1}
$$

when $n$ is large, where $Z_{1}$ is a random variable independent of all parameters following a Tracy-Widom distribution of type 1 .

This proposition describes the statistical distribution of the ratio (4.5) in the absence of a crack. As we will see, it allows us to compute explicitly the threshold of the likelihood-ratio test which is the most powerful test for a given false alarm rate by the Neyman-Pearson lemma.

The singular value distribution of the MSR matrix in the presence of crack. Now we turn to the case where the symmetrized MSR matrix $\mathbf{A}^{s}$ is obtained with a single crack. Recall that $\sigma_{0}$ is the nonzero singular value of $\mathbf{A}_{0}$ and $\sigma_{1}^{(n)} \geq \sigma_{2}^{(n)} \geq \sigma_{3}^{(n)} \geq \cdots \geq \sigma_{n}^{(n)}$ are the singular values of the matrix $\mathbf{A}^{s}$. For large $n$, we can expand the distribution of the singular values and we obtain the following results.

Proposition 4.3. When $n \rightarrow \infty$ :

a) If $\sigma_{\mathrm{c}}>\sigma_{0}$, then the largest singular value $\sigma_{1}^{(n)}$ obeys the same non-Gaussian statistics (4.3) as in the absence of the crack.

b) If $\sigma_{\mathrm{c}}<\sigma_{0}$, then the largest singular value $\sigma_{1}^{(n)}$ obeys Gaussian statistics with the mean and variance

$$
\mathbb{E}\left(\sigma_{1}^{(n)}\right)=\sigma_{0}+\sigma_{\mathrm{c}}^{2} \sigma_{0}^{-1}, \quad \operatorname{Var}\left(\sigma_{1}^{(n)}\right)=\frac{1}{n} \sigma_{\mathrm{c}}^{2}\left(1-\sigma_{\mathrm{c}}^{2} \sigma_{0}^{-2}\right)
$$

c) For any $\sigma_{\mathrm{c}}$ the second singular value $\sigma_{2}^{(n)}$ is equal to $2 \sigma_{\mathrm{c}}$ to leading order.

d) The normalized $l^{2}$-norm of the $n-1$ smallest singular values satisfies

$$
n\left[\frac{1}{n-1} \sum_{j=2}^{n}\left(\sigma_{j}^{(n)}\right)^{2}-\sigma_{\mathrm{c}}^{2}\right] \stackrel{\text { dist. }}{=} \mathcal{N}\left(2 \sigma_{\mathrm{c}}^{2}+\sigma_{0}^{2}-\bar{\sigma}_{1}^{2}, 2 \sigma_{\mathrm{c}}^{4}\right),
$$

where $\bar{\sigma}_{1}=2 \sigma_{\mathrm{c}}$ if $\sigma_{\mathrm{c}}>\sigma_{0}$ and $\bar{\sigma}_{1}=\sigma_{0} \sigma_{\mathrm{c}}^{2} \sigma_{0}^{-1}$ if $\sigma_{\mathrm{c}}<\sigma_{0}$. 
Proof. Points a) and b) are proved in [18]. Point c) is proved in [13]. Point d) can be proved by using the explicit representation of the $l^{2}$-norm of the singular values in terms of the entries of the matrix and the use of the central limit theorem.

Several interesting features can be observed:

- The noise generates many small singular values, whose largest one is $\sigma_{2}^{(n)}$ which is of the order of $2 \sigma_{\mathrm{c}}$.

- The first singular value, $\sigma_{1}^{(n)}$, corresponding to the crack, increases as the noise increases. This is a manifestation of the level repulsion well-known in random matrix theory [23]: the small singular values (and in particular $\sigma_{2}^{(n)}$ ) increase as the noise increases, and the strong singular value is repelled.

- The first singular value, corresponding to the crack, and the second singular value, that is the largest singular value generated by the noise, are well separated as long as $\mathbb{E}\left(\sigma_{1}^{(n)}\right)>\mathbb{E}\left(\sigma_{2}^{(n)}\right)$, i.e., $\sigma_{\mathrm{c}}<\sigma_{0}$. In the opposite case $\sigma_{\mathrm{c}}>\sigma_{0}$ it is not possible to see the crack from the singular values.

We can now describe the ratio of the maximal singular value over the normalized $l^{2}$-norm as follows.

Proposition 4.4. Let us consider the symmetrized MSR matrix obtained in the presence of measurement noise with a crack. For $\sigma_{\mathrm{c}}<\sigma_{0}$, the ratio $R$ defined by (4.5) has the following statistical distribution

$$
R \stackrel{\text { dist. }}{=} \frac{\sigma_{0}}{\sigma_{\mathrm{c}}}+\frac{\sigma_{\mathrm{c}}}{\sigma_{0}}+\frac{1}{\sqrt{n}} \sqrt{1-\sigma_{\mathrm{c}}^{2} \sigma_{0}^{-2}} \mathcal{N}(0,1) .
$$

For $\sigma_{\mathrm{c}}>\sigma_{0}$ we have (4.6).

This proposition describes the statistical distribution of the ratio (4.5) in the presence of a crack. It allows us to compute explicitly the power of the likelihoodratio test which is the most powerful test for a given false alarm rate by the NeymanPearson lemma.

5. Berens' Modelling Revisited. In [7] A.P. Berens introduced a framework for analyzing schemes for nondestructive inspection methods, testing for the presence of flaws. In this reliability analysis the probability of detection (POD) as function of flaw size played a central role. In our notation the "flaw size" corresponds to the parameter $\varepsilon_{\omega}$ and we are thus interested in designing reliability tests with a desirable performance in terms of the corresponding $\operatorname{POD}\left(\varepsilon_{\omega}\right)$ function. In [7] a maximum likelihood approach was used for parameter estimation, and a log normal distribution was in particular postulated for the response variable's relation to crack size. One parameter to be estimated is then the variance of the Gaussian residual. Our approach here is to introduce a physical model for the measurements, as we have described above, and then infer a corresponding "optimal" POD function that can be associated with the MSR matrix measurements. We describe the picture deriving from this approach below, in fact, the resulting picture deviates somewhat from that deriving from Berens' modelling.

Consider the imaging of cracks from measurements of the MSR matrix at a single frequency $\omega$ in the presence of measurement noise, that is, we model with an additive Gaussian noise. Assuming availability of previous and/or multiple measurements we may assume that the variance of the entries of the MSR matrix (due to the measurement noise) is known and equal to $a^{2}$. In fact, we will see that we do not need to know the value $a^{2}$ in order to build the most powerful test with a prescribed false alarm rate.

In the absence of the crack (hypothesis $H_{0}$ ) the ratio $R$ of the first singular value of the symmetrized MSR matrix over the normalized $l^{2}$-norm of the other singular values is of the form (4.6) by Proposition 4.2.

In the presence of a crack at position $\boldsymbol{x}_{c}$ and with size $l_{c}$ (hypothesis $H_{A}$ ), Proposition 4.4 shows that the ratio is of the form (4.7). This result is correct as 
long as $\sigma_{0}>\sigma_{\mathrm{c}}$. When $\sigma_{0}<\sigma_{\mathrm{c}}$ we have (4.6).

If the data gives the ratio $R$, then we propose to use a test of the form $R>r$ for the alarm corresponding to the presence of a crack. By the Neyman-Pearson lemma (see Appendix A) the decision rule of accepting $H_{A}$ if and only if $R>r_{\alpha}$ maximizes the probability of detection for a given false alarm probability $\alpha$

$$
\alpha=\mathbb{P}\left(R>r_{\alpha} \mid H_{0}\right)
$$

with the threshold

$$
r_{\alpha}=2+\frac{1}{2^{2 / 3} n^{2 / 3}} \Phi_{\mathrm{TW} 1}^{-1}(1-\alpha),
$$

where $\Phi_{\mathrm{TW} 1}$ is the cumulative distribution function of the Tracy-Widom distribution of type 1. The computation of the threshold is easy since it depends only on the number of sensors $n$ and on the false alarm probability $\alpha$. This test is therefore universal. Note that we should use a Tracy-Widom distribution table, and not a Gaussian table. We have, for instance, $\Phi_{\mathrm{TW} 1}^{-1}(0.9) \simeq 0.45, \Phi_{\mathrm{TW} 1}^{-1}(0.95) \simeq 0.98$, and $\Phi_{\mathrm{TW} 1}^{-1}(0.99) \simeq 2.02$.

The detection probability $1-\beta$ is the probability to sound the alarm when there is a crack:

$$
1-\beta=\mathbb{P}\left(R>r_{\alpha} \mid H_{A}\right) .
$$

For a given measurement array it depends on $l_{c}$ and $\boldsymbol{x}_{c}$ through the value $\sigma_{0}\left(l_{c}, \boldsymbol{x}_{c}\right)$ and also on the noise level $a$. Here we find that the detection probability is

$$
\operatorname{POD}\left(l_{c}, \boldsymbol{x}_{c}\right)=1-\beta\left(l_{c}, \boldsymbol{x}_{c}\right)=\Phi\left(\sqrt{n} \frac{\frac{\sigma_{0}}{\sigma_{c}}+\frac{\sigma_{c}}{\sigma_{0}}-r_{\alpha}}{\sqrt{1-\left(\sigma_{c} / \sigma_{0}\right)^{2}}}\right),
$$

where $\Phi$ is the cumulative distribution function of the normal distribution with mean zero and variance one. This result is valid as long as $\sigma_{0}>\sigma_{\mathrm{c}}$. When $\sigma_{0}<\sigma_{\mathrm{c}}$, so that the crack is "hidden in noise", then we have $1-\beta=1-\Phi_{\mathrm{TW} 1}\left(\Phi_{\mathrm{TW} 1}^{-1}(1-\alpha)\right)=\alpha$. Note that, as functions of the number of sensors $n$, the singular value $\sigma_{0}$ scales as $n$, while the noise level $\sigma_{\mathrm{c}}$ scales as $\sqrt{n}$. This shows that the detection power increases with the number of sensors.

6. Conditional Localization. We assume that the spectral test described in Section 5 identified the presence of a crack and we want to estimate its location. Thus, we want to decide whether there is a crack or not at a particular location based on the measured MSR matrix, $\mathbf{A}$, at a single or for multiple frequencies.

6.1. Effective Imaging Functional. We shall assume that there is one crack present as determined by the spectral detection test described above and seek to estimate the location $\boldsymbol{x}_{c}$ of the crack. We model the observations as

$$
\mathbf{A}^{s}(\omega)=\tau(\omega, \boldsymbol{x}) \boldsymbol{d}(\omega, \boldsymbol{x}) \boldsymbol{d}(\omega, \boldsymbol{x})^{T}+\mathbf{W}^{s}(\omega)
$$

in order to specify the conditional distribution of the data $\mathbf{A}^{s}$ given the parameters $\boldsymbol{x}, \tau$, and $a$. In this case, the observed symmetrized MSR matrix $\mathbf{A}^{s}(\omega)$ has the probability density function

$$
p\left(\mathbf{A}^{s} \mid \boldsymbol{x}, \tau, a\right)=\frac{1}{2^{n} \pi^{\left(n^{2}+n\right) / 2} a^{n^{2}+n}} \exp \left(-\frac{\left\|\mathbf{A}^{s}-\tau \boldsymbol{d}(\omega, \boldsymbol{x}) \boldsymbol{d}(\omega, \boldsymbol{x})^{T}\right\|_{F}^{2}}{2 a^{2}}\right),
$$

with respect to the measure over the space of complex symmetric matrices:

$$
\prod_{j=1}^{n} d \Re\left(A_{j, j}^{s}\right) d \Im\left(A_{j, j}^{s}\right) \prod_{1 \leq j<l \leq n} d \Re\left(A_{j, l}^{s}\right) d \Im\left(A_{j, l}^{s}\right) .
$$


Here the subscript $F$ represents the Frobenius norm.

Next, we find the conditional distribution of the parameters given the observations $\mathbf{A}^{s}$. The prior distribution is the uniform distribution for the parameter $\boldsymbol{x}$ over the search window and the Jeffreys prior for the parameters $\tau, a$ (a non-informative prior distribution that is proportional to the square root of the determinant of the Fisher information matrix), which is proportional to $a^{-1}$ [24, Section 2.7.1]. Using Bayes theorem, we find that the likelihood function of the parameters $\boldsymbol{x}, \tau$, and $a$ is given by

$$
l_{0}\left(\boldsymbol{x}, \tau, a \mid \mathbf{A}^{s}\right)=\frac{1}{a^{n^{2}+n+1}} \exp \left(-\frac{\left\|\mathbf{A}^{s}-\tau \boldsymbol{d}(\omega, \boldsymbol{x}) \boldsymbol{d}(\omega, \boldsymbol{x})^{T}\right\|_{F}^{2}}{2 a^{2}}\right) .
$$

The maximum likelihood estimator of $\boldsymbol{x}_{c}$ and the nuisance parameters $a$ and $\tau$ are then found by maximizing the likelihood function (6.1) with respect to these:

$$
(\hat{\boldsymbol{x}}, \hat{\tau}, \hat{a})=\arg \max _{\boldsymbol{x}, \tau, a} l_{0}\left(\boldsymbol{x}, \tau, a \mid \mathbf{A}^{s}\right) .
$$

We first eliminate $a$ by requiring

$$
\left.\frac{\partial l_{0}\left(\boldsymbol{x}, \tau, a \mid \mathbf{A}^{s}\right)}{\partial a}\right|_{a=\hat{a}}=0,
$$

which gives

$$
\hat{a}=\frac{\left\|\mathbf{A}^{s}-\tau \boldsymbol{d}(\omega, \boldsymbol{x}) \boldsymbol{d}(\omega, \boldsymbol{x})^{T}\right\|_{F}}{\sqrt{n^{2}+n+1}},
$$

and then the likelihood ratio is proportional to

$$
l_{0}\left(\boldsymbol{x}, \tau, \hat{a} \mid \mathbf{A}^{s}\right) \simeq\left\|\mathbf{A}^{s}-\tau \boldsymbol{d}(\omega, \boldsymbol{x}) \boldsymbol{d}(\omega, \boldsymbol{x})^{T}\right\|_{F}^{-\left(n^{2}+n+1\right) / 2} .
$$

Since $\mathbf{A}^{s}(\omega)$ is complex symmetric, it admits a symmetric singular value decomposition: there exist unitary vectors $\boldsymbol{u}_{j}(\omega)$ such that

$$
\mathbf{A}^{s}(\omega)=\sum_{j=1}^{n} \sigma_{j}^{(n)}(\omega) \boldsymbol{u}_{j}(\omega) \boldsymbol{u}_{j}(\omega)^{T}
$$

Therefore, we can write

$$
\left\|\mathbf{A}^{s}-\tau \boldsymbol{d}(\omega, \boldsymbol{x}) \boldsymbol{d}(\omega, \boldsymbol{x})^{T}\right\|_{F}^{2}=\left\|\boldsymbol{u}^{(2)}(\omega)-\tau \boldsymbol{d}^{(2)}(\omega, \boldsymbol{x})\right\|_{2}^{2},
$$

with $\boldsymbol{u}^{(2)}=\sum_{j=1}^{n} \sigma_{j}^{(n)} \boldsymbol{u}_{j} \otimes \boldsymbol{u}_{j}$ and $\boldsymbol{d}^{(2)}=\boldsymbol{d} \otimes \boldsymbol{d}$. Since $\|\boldsymbol{d}\|_{2}=1$, we have $\left\|\boldsymbol{d}^{(2)}\right\|_{2}=1$ and we then find that

$$
\hat{\tau}=\arg \min _{\tau}\left\|\boldsymbol{u}^{(2)}(\omega)-\tau \boldsymbol{d}^{(2)}(\omega, \boldsymbol{x})\right\|_{2}^{2}=\left(\boldsymbol{d}^{(2)}(\omega, \boldsymbol{x}), \boldsymbol{u}^{(2)}(\omega)\right) .
$$

Note also that $\left\|\boldsymbol{u}^{(2)}\right\|_{2}^{2}=\sum_{j=1}^{n}\left(\sigma_{j}^{(n)}\right)^{2}=\left\|\mathbf{A}^{s}\right\|_{F}^{2}$. We therefore conclude that the maximum likelihood estimator $\hat{\boldsymbol{x}}$ derives from maximizing the MUSIC-type function

$$
\hat{\boldsymbol{x}}=\arg \min _{\boldsymbol{x}}\left\|\boldsymbol{u}^{(2)}(\omega)-\left(\boldsymbol{d}^{(2)}(\omega, \boldsymbol{x}), \boldsymbol{u}^{(2)}(\omega)\right) \boldsymbol{d}^{(2)}(\omega, \boldsymbol{x})\right\|_{2}^{2} .
$$

Note however that $\hat{\boldsymbol{x}}$ is not the maximizer of the MUSIC functional (3.5) since all singular vectors (weighted by the singular values) contribute to $\boldsymbol{u}^{(2)}$. We have in fact

$$
\begin{aligned}
\left\|\boldsymbol{u}^{(2)}(\omega)-\left(\boldsymbol{d}^{(2)}(\omega, \boldsymbol{x}), \boldsymbol{u}^{(2)}(\omega)\right) \boldsymbol{d}^{(2)}(\omega, \boldsymbol{x})\right\|_{2}^{2} & =\left\|\boldsymbol{u}^{(2)}(\omega)\right\|_{2}^{2}-\left|\left(\boldsymbol{u}^{(2)}(\omega), \boldsymbol{d}^{(2)}(\omega, \boldsymbol{x})\right)\right|^{2} \\
& =\left\|\mathbf{A}^{s}(\omega)\right\|_{F}^{2}-\left|\mathcal{I}_{\mathrm{KM}}(\omega, \boldsymbol{x})\right|^{2}
\end{aligned}
$$


where $\mathcal{I}_{K M}$ is the Kirchhoff migration functional (3.6). From this representation we find that the estimation $\hat{\boldsymbol{x}}$ of the location $\boldsymbol{x}_{c}$ can be expressed in terms of the Kirchhoff migration functional as

$$
\hat{\boldsymbol{x}}=\arg \max _{\boldsymbol{x}}\left|\mathcal{I}_{\mathrm{KM}}(\omega, \boldsymbol{x})\right| .
$$

This indicates that Kirchhoff migration is more accurate in the presence of additive noise than MUSIC in that the location of its maximum is exactly the maximum likelihood estimator of the location of the crack.

The analysis can be extended to the case in which the MSR matrices are recorded at several frequencies $\left(\omega_{k}\right)_{k=1, \ldots, K}$ and the additive noise matrices $\mathbf{W}\left(\omega_{k}\right)$, $k=1, \ldots, K$, are independent and identically distributed. Then one finds that the maximum likelihood estimator of the location of the crack is the maximum of the matched field imaging functional:

$$
\hat{\boldsymbol{x}}=\arg \max _{\boldsymbol{x}} \mathcal{I}_{\mathrm{MF}}(\boldsymbol{x}),
$$

where $\mathcal{I}_{M F}$ is the Matched Field functional (3.11). This shows that one should look for the maximum of the sum of the square moduli of the KM functionals in order to exploit the multi-frequency information optimally. Indeed, the fact that the relevant operation is the sum of the squares comes from the fact that the additive noise matrices are assumed to be independent for different frequencies. If some correlation between frequency components exists, it is likely that a procedure such as Coherent Interferometry [8] will be more appropriate.

To conclude this section, we remark on the case when there are several cracks. We can then use a recursively applied and projected version of the approach above. Let $\Pi_{k}^{\perp}$ denote the unitary projection matrix on the complement of the subspace spanned by the illuminated vectors $\boldsymbol{d}\left(\omega, \hat{\boldsymbol{x}}_{j}\right), j=1, \ldots, k$ of the first $k$ estimated crack locations. We then form the modified MSR matrix by projecting as:

$$
\mathbf{A}_{k}^{s}(\omega)=\Pi_{k}^{\perp} \mathbf{A}^{s}(\omega) \Pi_{k}^{\perp} .
$$

The test for presence of further cracks is then carried out as described in Section 5 , but with respect to the projected MSR. In the case that the test predicts that further cracks are present then the subsequent crack location is estimated by again maximizing a Kirchhoff imaging functional $\mathcal{I}_{\mathrm{KM}, k}$, associated with the projected $\operatorname{MSR} \mathbf{A}_{k}^{s}$.

We remark that above we assumed that the cracks were well-resolved so that the corresponding illumination vectors are orthogonal. In the case that clusters of nearby cracks are present one can generalize the above approach by postprocessing the data such that the location estimates for the cracks in the cluster is estimated by maximizing the joint likelihood of the cracks.

6.2. Statistical Analysis of Location Estimate. We continue here the analysis of the single frequency case and provide a location-dependent threshold for the image function that we derived above. We remark that this is important since the detection test introduced in Section 4 only tests whether there is something present in the probed scenery or not. Here we want to test whether there is a localized crack present at the particular search location $\boldsymbol{x}^{S}$. As a part of this test we will then obtain a measure of confidence with which we can say that there is a crack present.

We choose as a test statistics the image function derived above

$$
\mathcal{I}_{\mathrm{KM}}\left(\omega, \boldsymbol{x}^{S}\right)=\left(\boldsymbol{d}\left(\omega, \boldsymbol{x}^{S}\right), \mathbf{A}^{s}(\omega) \overline{\boldsymbol{d}\left(\omega, \boldsymbol{x}^{S}\right)}\right)=\left(\boldsymbol{d}\left(\omega, \boldsymbol{x}^{S}\right), \mathbf{A}(\omega) \overline{\boldsymbol{d}\left(\omega, \boldsymbol{x}^{S}\right)}\right) .
$$


We observe that under the null hypothesis $H_{0}$, there is no crack at $\boldsymbol{x}^{S}$, we have

$$
\mathcal{I}_{\mathrm{KM}}\left(\omega, \boldsymbol{x}^{S}\right) \stackrel{\text { dist. }}{\simeq} \frac{a}{\sqrt{2}}\left(W_{1}+i W_{2}\right),
$$

with $W_{j}$ being standard independent normal real Gaussian random variables with mean zero and variance one. Here $a^{2}$ is the variance of the entries of the random matrix $\mathbf{A}$ and we have implicitly assumed that $\boldsymbol{x}^{S}$ is far enough from the other cracks so that the vector $\boldsymbol{d}\left(\omega, \boldsymbol{x}^{S}\right)$ is approximately orthogonal to the illumination vectors of other cracks. Under the alternate hypothesis $H_{A}$, we have

$$
\mathcal{I}_{\mathrm{KM}}\left(\omega, \boldsymbol{x}^{S}\right) \stackrel{\text { dist. }}{\simeq} \mu_{\mathrm{KM}}\left(\omega, \boldsymbol{x}^{S}\right)+\frac{a}{\sqrt{2}}\left(W_{1}+i W_{2}\right),
$$

where $\mu_{\mathrm{KM}}\left(\omega, \boldsymbol{x}^{S}\right)$ is given by

$$
\mu_{\mathrm{KM}}\left(\omega, \boldsymbol{x}^{S}\right)=\tau\left(\omega, \boldsymbol{x}^{S}\right)\left|\boldsymbol{d}\left(\omega, \boldsymbol{x}^{S}\right)\right|^{4}=\tau\left(\omega, \boldsymbol{x}^{S}\right) .
$$

Here $\tau$ given by (3.3) with $\boldsymbol{x}_{c}$ replaced by $\boldsymbol{x}^{S}$.

We now consider the real and imaginary parts of the statistics:

$$
y_{1}=\Re\left(\mathcal{I}_{\mathrm{KM}}\left(\omega, \boldsymbol{x}^{S}\right)\right), \quad y_{2}=\Im\left(\mathcal{I}_{\mathrm{KM}}\left(\omega, \boldsymbol{x}^{S}\right)\right),
$$

and we denote similarly $\mu_{1}=\Re\left(\mu_{\mathrm{KM}}\left(\omega, \boldsymbol{x}^{S}\right)\right)$ and $\mu_{2}=\Im\left(\mu_{\mathrm{KM}}\left(\omega, \boldsymbol{x}^{S}\right)\right)$. From the expression (3.3) of $\tau\left(\omega, \boldsymbol{x}^{S}\right)$, it is clear that its argument is very close to $\pi / 2$ so that $\mu_{2}$ is positive and larger than $\mu_{1}$ and the relevant information is in the imaginary part of the imaging functional $y_{2}=\Im\left(\mathcal{I}_{\mathrm{KM}}\left(\omega, \boldsymbol{x}^{S}\right)\right)$. Using the expression of the normal density we find that the likelihood ratio for $y_{2}$ is given by

$$
\Lambda\left(y_{2}\right)=\frac{f_{A}\left(y_{2}\right)}{f_{0}\left(y_{2}\right)}=\exp \left(-\frac{\mu_{2}^{2}}{a^{2}}+\frac{2 \mu_{2} y_{2}}{a^{2}}\right) .
$$

By the Neyman-Pearson lemma the decision rule of accepting $H_{A}$ if and only if $y_{2}=\Im\left(\mathcal{I}_{\mathrm{KM}}\left(\omega, \boldsymbol{x}^{S}\right)\right)>\eta$ maximizes the probability of detection for a given false alarm rate $\alpha$ with the threshold $\eta=(a / \sqrt{2}) \Phi^{-1}(1-\alpha)$, where $\Phi$ is the cumulative distribution function of the normal distribution. The power of the test is given by

$$
1-\beta_{\mathrm{KM}}\left(\omega, \boldsymbol{x}^{S}\right)=\Phi\left(\sqrt{2} \frac{\Im\left(\mu_{\mathrm{KM}}\left(\omega, \boldsymbol{x}^{S}\right)\right)-\eta}{a}\right) .
$$

The power of the test can be expressed in terms of the SNR

$$
\operatorname{SNR}\left(\omega, \boldsymbol{x}^{S}\right):=\frac{\left|\mathbb{E}\left(\mathcal{I}_{\mathrm{KM}}\left(\omega, \boldsymbol{x}^{S}\right)\right)\right|}{\operatorname{Var}^{1 / 2}\left(\mathcal{I}_{\mathrm{KM}}\left(\omega, \boldsymbol{x}^{S}\right)\right)}=\frac{\left|\tau\left(\omega, \boldsymbol{x}^{S}\right)\right|}{a},
$$

as

$$
1-\beta_{\mathrm{KM}}\left(\omega, \boldsymbol{x}^{S}\right)=\Phi\left(\sqrt{2} \operatorname{SNR}\left(\omega, \boldsymbol{x}^{S}\right)-\Phi^{-1}(1-\alpha)\right),
$$

where we have made the approximation $\left|\mu_{\mathrm{KM}}\left(\omega, \boldsymbol{x}^{S}\right)\right| \simeq \Im\left(\mu_{\mathrm{KM}}\left(\omega, \boldsymbol{x}^{S}\right)\right)$ since the argument of $\tau\left(\omega, \boldsymbol{x}^{S}\right)$ is close to $\pi / 2$. Note that the order of magnitude of the SNR is

$$
\operatorname{SNR}\left(\omega_{0}, \boldsymbol{x}^{S}\right) \simeq \operatorname{SNR}_{0}:=\frac{c_{0} n}{4 \omega_{0} L\left|\ln \left(\frac{\varepsilon_{\omega_{0}}}{4}\right)+\gamma-\frac{i \pi}{2}\right|},
$$

where $\omega_{0}$ is the typical frequency and $L$ is the distance from the sensor array to the search region. 
6.3. Multifrequency Measurements. In the case of (uncorrelated) measurements at multiple frequencies, one can use the migration imaging functional (3.9) which can be written as

$$
\mathcal{I}_{\mathrm{KMF}}\left(\boldsymbol{x}^{S}\right)=\frac{1}{K} \sum_{\omega_{k}} \mathcal{I}_{\mathrm{KM}}\left(\omega_{k}, \boldsymbol{x}^{S}\right)
$$

where $K$ is the number of frequencies. Under the null hypothesis $H_{0}$, there is no crack at $\boldsymbol{x}^{S}$, the functional $\mathcal{I}_{\mathrm{KMF}}\left(\boldsymbol{x}^{S}\right)$ is distributed as

$$
\mathcal{I}_{\mathrm{KMF}}\left(\boldsymbol{x}^{S}\right) \stackrel{\text { dist. }}{\simeq} \frac{a}{\sqrt{2 K}}\left(W_{1}+i W_{2}\right)
$$

with again $W_{j}$ being standard independent normal real Gaussian random variables with mean zero and variance one. Under the alternate hypothesis $H_{A}$, we have

$$
\mathcal{I}_{\mathrm{KMF}}\left(\boldsymbol{x}^{S}\right) \stackrel{\text { dist. }}{\simeq} \mu_{\mathrm{KMF}}\left(\boldsymbol{x}^{S}\right)+\frac{a}{\sqrt{2 K}}\left(W_{1}+i W_{2}\right),
$$

where $\mu_{\mathrm{KMF}}\left(\boldsymbol{x}^{S}\right)$ is given by

$$
\mu_{\mathrm{KMF}}\left(\boldsymbol{x}^{S}\right)=\frac{1}{K} \sum_{\omega_{k}} \mu_{\mathrm{KM}}\left(\omega_{k}, \boldsymbol{x}^{S}\right) .
$$

A straightforward generalization of the arguments used in the previous subsection shows that the test accepting $H_{A}$ if $\Im\left(\mathcal{I}_{\mathrm{KMF}}\left(\boldsymbol{x}^{S}\right)\right)>\eta$ maximizes the probability of detection for a given false alarm rate $\alpha$ with the threshold $\eta=(a / \sqrt{2 K}) \Phi^{-1}(1-\alpha)$. The power of this test is

$$
1-\beta_{\mathrm{KMF}}\left(\boldsymbol{x}^{S}\right)=\Phi\left(\sqrt{2 K} \frac{\Im\left(\mu_{\mathrm{KMF}}\left(\boldsymbol{x}^{S}\right)\right)-\eta}{a}\right) \simeq \Phi\left(\sqrt{2 K} \mathrm{SNR}_{0}-\Phi^{-1}(1-\alpha)\right),
$$

with $\mathrm{SNR}_{0}$ defined by (6.7). Therefore, the multiple frequencies enhance the detection performance via higher "effective" SNR.

One can also use the matched field imaging functional (3.11)

$$
\mathcal{I}_{\mathrm{MF}}\left(\boldsymbol{x}^{S}\right)=\frac{1}{K} \sum_{\omega_{k}}\left|\mathcal{I}_{\mathrm{KM}}\left(\omega_{k}, \boldsymbol{x}^{S}\right)\right|^{2} .
$$

Under the null hypothesis $H_{0}$, there is no crack at $\boldsymbol{x}^{S}$, the distribution of the functional $\mathcal{I}_{\mathrm{MF}}\left(\boldsymbol{x}^{S}\right)$ is proportional to a $\chi^{2}$-distribution with $2 K$ degrees of freedom and it can be approximated when $K$ is large enough $(2 K>50$ in practice) by

$$
\mathcal{I}_{\mathrm{MF}}\left(\boldsymbol{x}^{S}\right) \stackrel{\text { dist. }}{\simeq} a^{2}+\frac{a^{2}}{\sqrt{K}} \mathcal{N}(0,1) \text {. }
$$

Under the alternate hypothesis $H_{A}$, we have for $K$ large enough

$$
\mathcal{I}_{\mathrm{MF}}\left(\boldsymbol{x}^{S}\right) \stackrel{\text { dist. }}{\simeq} a^{2}+\mu_{\mathrm{MF}}^{2}\left(\boldsymbol{x}^{S}\right)+\frac{a}{\sqrt{K}} \sqrt{2 \mu_{\mathrm{MF}}^{2}\left(\boldsymbol{x}^{S}\right)+a^{2}} \mathcal{N}(0,1),
$$

where $\mu_{\mathrm{MF}}\left(\boldsymbol{x}^{S}\right)$ is given by

$$
\mu_{\mathrm{MF}}^{2}\left(\boldsymbol{x}^{S}\right)=\frac{1}{K} \sum_{\omega_{k}}\left|\mu_{\mathrm{KM}}\left(\omega_{k}, \boldsymbol{x}^{S}\right)\right|^{2}=\frac{1}{K} \sum_{\omega_{k}}\left|\tau\left(\omega_{k}, \boldsymbol{x}^{S}\right)\right|^{2} .
$$

The test accepting $H_{A}$ if $\mathcal{I}_{\mathrm{MF}}\left(\boldsymbol{x}^{S}\right)>\eta$ maximizes the probability of detection for a given false alarm rate $\alpha$ with the threshold

$$
\eta=a^{2}+\frac{a^{2}}{\sqrt{K}} \Phi^{-1}(1-\alpha) .
$$


The power of this test is

$$
1-\beta_{\mathrm{MF}}\left(\boldsymbol{x}^{S}\right)=\Phi\left(\sqrt{K} \frac{\mu_{\mathrm{MF}}^{2}\left(\boldsymbol{x}^{S}\right)}{a \sqrt{a^{2}+2 \mu_{\mathrm{MF}}^{2}\left(\boldsymbol{x}^{S}\right)}}-\frac{a}{\sqrt{a^{2}+2 \mu_{\mathrm{MF}}^{2}\left(\boldsymbol{x}^{S}\right)}} \Phi^{-1}(1-\alpha)\right) .
$$

If the SNR is larger than one, then the power is

$$
1-\beta_{\mathrm{MF}}\left(\boldsymbol{x}^{S}\right)=\Phi\left(\frac{\sqrt{K}}{\sqrt{2}} \mathrm{SNR}_{0}-\mathrm{SNR}_{0}^{-1} \Phi^{-1}(1-\alpha)\right) .
$$

If the SNR is smaller than one, then the power is

$$
1-\beta_{\mathrm{MF}}\left(\boldsymbol{x}^{S}\right)=\Phi\left(\sqrt{K} \mathrm{SNR}_{0}^{2}-\Phi^{-1}(1-\alpha)\right)
$$

This shows that the power is smaller than with the test using the KMF functional.

7. Identification of Crack Orientation. Assume that one has detected a crack. The location of the crack $\boldsymbol{x}_{c}$ can be estimated by $\hat{\boldsymbol{x}}$ given by (6.2). Using the expression (3.3) of the first singular value $\sigma_{1}^{(n)}=\left|\tau\left(\omega, \boldsymbol{x}_{c}\right)\right|$ of the MSR matrix we obtain an estimate of the length $\hat{l}$ of the crack:

$$
\gamma+\ln \frac{\omega \hat{l}}{4 c_{0}}=\left(\frac{c_{0}^{2}}{16 \omega^{2}\left(\sigma_{1}^{(n)}\right)^{2}}\left(\sum_{j=1}^{n} \frac{1}{\left|\boldsymbol{x}_{j}-\hat{\boldsymbol{x}}\right|}\right)^{2}-\frac{\pi^{2}}{4}\right)^{1 / 2} .
$$

Here we seek to estimate the orientation $\boldsymbol{t}_{c}$ of the crack. We have from (2.4)

$$
\left|A_{j l}(\omega)\right|^{2} \simeq A_{j l}^{(0)}(\omega)+A_{j l}^{(1)}(\omega)\left(\boldsymbol{n}_{j} \cdot \boldsymbol{t}_{c}\right)\left(\boldsymbol{n}_{l} \cdot \boldsymbol{t}_{c}\right)
$$

where

$$
\begin{aligned}
& A_{j l}^{(0)}(\omega)=\frac{c_{0}^{2}}{16 \omega^{2}\left|\boldsymbol{x}_{j}-\boldsymbol{x}_{\mathrm{c}}\right|\left|\boldsymbol{x}_{l}-\boldsymbol{x}_{\mathrm{c}}\right|} \frac{1}{\left|\ln \left(\frac{\varepsilon_{\omega}}{4}\right)+\gamma-\frac{i \pi}{2}\right|^{2}}, \\
& A_{j l}^{(1)}(\omega)=-\frac{\varepsilon_{\omega}^{2}}{16 \omega^{2}\left|\boldsymbol{x}_{j}-\boldsymbol{x}_{\mathrm{c}}\right|\left|\boldsymbol{x}_{l}-\boldsymbol{x}_{\mathrm{c}}\right|} \Re\left(\frac{1}{\ln \left(\frac{\varepsilon_{\omega}}{4}\right)+\gamma-\frac{i \pi}{2}}\right),
\end{aligned}
$$

$\boldsymbol{n}_{j}=\left(\boldsymbol{x}_{c}-\boldsymbol{x}_{j}\right) /\left|\boldsymbol{x}_{c}-\boldsymbol{x}_{j}\right|$, and $\varepsilon_{\omega}=\omega l_{c} / c_{0}$. Since $A_{j l}^{(0)}(\omega)$ and $A_{j l}^{(1)}(\omega)$ can be estimated by their respective expressions $\hat{A}_{j l}^{(0)}(\omega)$ and $\hat{A}_{j l}^{(1)}(\omega)$ in terms of the estimates $\hat{\boldsymbol{x}}$ of $\boldsymbol{x}_{\mathrm{c}}$ and $\hat{l}$ of $l_{c}$, the procedure for estimation of $\boldsymbol{t}$ exploits the $(j, l, \omega)$-dependence of $\left|A_{j l}(\omega)\right|^{2}$ as follows:

$$
\hat{t}=\operatorname{argmin}_{\boldsymbol{t}} \mathcal{R}(\boldsymbol{t})
$$

where

$$
\mathcal{R}(\boldsymbol{t})=\left.\sum_{j, l, \omega_{k}}|| A_{j l}\left(\omega_{k}\right)\right|^{2}-\hat{A}_{j l}^{(0)}\left(\omega_{k}\right)-\left.\hat{A}_{j l}^{(1)}\left(\omega_{k}\right)\left(\boldsymbol{n}_{j} \cdot \boldsymbol{t}\right)\left(\boldsymbol{n}_{l} \cdot \boldsymbol{t}\right)\right|^{2} .
$$

To conclude this section, we make one remark. It is possible to design a coherent estimator by looking at the mismatch between $A_{j l}(\omega)$ and the theoretical expression (2.4). However, this least-square problem is very sensitive to the estimate of the location of the crack through the phase term of (2.4). Therefore, we have preferred to introduce an incoherent estimator based on the mismatch between $\left|A_{j l}(\omega)\right|^{2}$ and its theoretical expression that is much more robust. 
8. Numerical Experiments. This section presents results of numerical experiments that give qualitative illustrations of some of the main findings in this paper. We choose the two following cracks:

$$
\Sigma_{j}=\left\{\boldsymbol{x}_{c, j}+x \boldsymbol{t}_{c, j},-l_{c} \leq x \leq l_{c}\right\}, \quad j=1,2,
$$

where $\boldsymbol{x}_{c, 1}=(0.2,-11.5), \boldsymbol{t}_{c, 1}=(1,0), \boldsymbol{x}_{c, 2}=(-0.4,-10.5), \boldsymbol{t}_{c, 2}=(\cos (\pi / 6), \sin (\pi / 6))$, and $l_{c}=0.01$. Here we set $c_{0}=2 \pi$ (so that $\varepsilon_{\omega}=0.5$ for $\omega=314$ ) and suppose that the transducers are equidistributed on the line going from $\left(x_{1}^{(1)}, 0\right)$ to $\left(x_{1}^{(n)}, 0\right)$.

8.1. Imaging Functionals. First, we consider imaging of a single crack using the functionals $\mathcal{I}_{\mathrm{MU}}, \mathcal{I}_{\mathrm{KM}}, \mathcal{I}_{\mathrm{KMF}}$, and $\mathcal{I}_{\mathrm{MT}}$ to illustrate their performance and limitations. Recall that these are the "MUSIC", "Kirchhoff", "Multifrequency Kirchhoff" and "One Mode Kirchhoff" given in respectively (3.5), (3.6), (3.9) and (3.10) in the situation with a crack. The test configurations are given in Table 8.1. Note that the set of data is generated by numerically solving the forward problem, that is solving (2.1), using an integral equation code.

\begin{tabular}{|c|c|c|c|c|c|c|}
\hline Crack & $n$ & $x_{1}^{(1)}$ & $x_{1}^{(n)}$ & Frequency & Imaging functional & Figure \\
\hline$\Sigma_{1}$ & 20 & -1 & 1 & $\omega \in[300,360]$ & $\mathcal{I}_{M T}, \mathcal{I}_{K M F}$ & Figs. 8.1, 8.4 \\
\hline$\Sigma_{2}$ & 20 & -1 & 1 & $\omega \in[300,360]$ & $\mathcal{I}_{M T}, \mathcal{I}_{K M F}$ & Fig. 8.2 \\
\hline$\Sigma_{1}$ & 20 & -1 & 1 & $\omega=330$ & $\mathcal{I}_{K M}, \mathcal{I}_{M U}$ & Figs. 8.1, 8.4 \\
\hline$\Sigma_{2}$ & 20 & -1 & 1 & $\omega=330$ & $\mathcal{I}_{K M}, \mathcal{I}_{M U}$ & Fig. 8.2 \\
\hline$\Sigma_{1}$ & 20 & -1 & 1 & $\begin{array}{r}\omega=300,320, \\
340,360\end{array}$ & $\mathcal{I}_{M U}$ & Fig. 8.3 \\
\hline
\end{tabular}

Test configurations for Figures 8.1-8.4.

The interval $[300,360]$ is uniformly partitioned into $K=60$ frequencies. The discretization size of the search domain is chosen as 0.05. Localization results for $\Sigma_{1}$ and $\Sigma_{2}$ are shown in Figs. 8.1 and 8.2, respectively. The locations of $\Sigma_{1}$ and $\Sigma_{2}$ are successfully identified, but we can observe that Kirchhoff Migration and MUSIC have poor range resolution, since they use only a single frequency.

Fig. 8.3 shows that the performance of the MUSIC algorithm over the considered range of frequencies $[300,360]$ is almost invariant. In the the other examples, we shall use the middle frequency, $\omega=330$, for this single-frequency algorithm.

Let us stress that using data computed by the asymptotic expansion formula (2.4) yields closely resembling images. In Fig. 8.4, localization results for $\Sigma_{1}$ using the asymptotic expansion formula are shown. That the imaging functionals are efficient when the forward problem is computed with the asymptotic formulas is indeed expected as they have been constructed via this representation. The comparisons between Fig. 8.4 and Fig. 8.1, however, show that the imaging functionals are efficient as well when the forward data is generated by solving (2.1) numerically, giving data which reflects more closely a situation with "real data".

8.1.1. Influence of the Transducer Array Setting. Next, we show the influence of the configuration of the transducer array with the test configurations being described in Table 8.2. The used imaging functional is $\mathcal{I}_{\mathrm{MT}}$.

In Fig. 8.5, we can see that for a given number of transducers, if the size of the array is small then the images are blurred (setting 1 ) while if it is large, then an aliasing effect due to undersampling of the array can appear (setting 4).

8.1.2. Estimation of the Tangential Direction of a Crack. We now estimate the tangential direction $\boldsymbol{t}$ of a single crack. Based on formula (7.1), the 

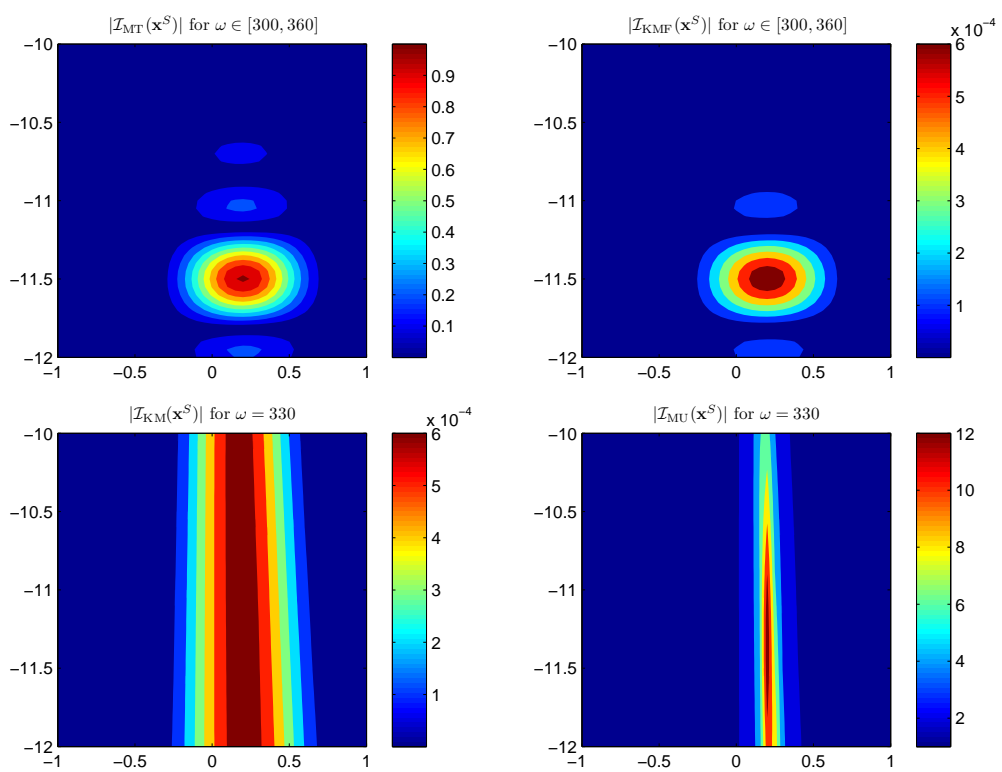

FIG. 8.1. Localization results for $\Sigma_{1}$ using $\mathcal{I}_{\mathrm{MT}}$ (top left), $\mathcal{I}_{\mathrm{KMF}}$ (top right), $\mathcal{I}_{\mathrm{KM}}$ (bottom left) and $\mathcal{I}_{\mathrm{MU}}$ (bottom right). The data set is generated solving (2.1) numerically.
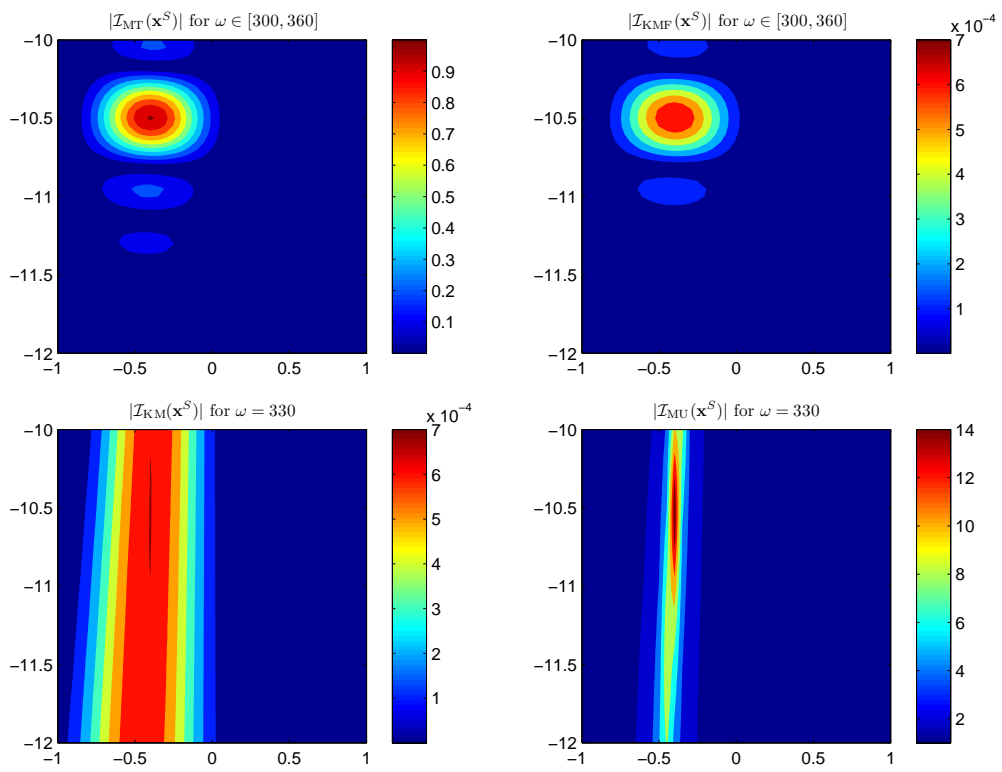

FIG. 8.2. Localization results for $\Sigma_{2}$.

\begin{tabular}{|c|c|c|c|c|c|c|}
\hline Crack & $n$ & $x_{1}^{(1)}$ & $x_{1}^{(n)}$ & Frequency & Imaging functional & Setting \\
\hline$\Sigma_{2}$ & 20 & -0.5 & 0.5 & $\omega \in[300,360]$ & $\mathcal{I}_{\mathrm{MT}}$ & setting 1 \\
\hline$\Sigma_{2}$ & 20 & -1 & 1 & $\omega \in[300,360]$ & $\mathcal{I}_{\mathrm{MT}}$ & setting 2 \\
\hline$\Sigma_{2}$ & 20 & -5 & 5 & $\omega \in[300,360]$ & $\mathcal{I}_{\mathrm{MT}}$ & setting 3 \\
\hline$\Sigma_{2}$ & 20 & -10 & 10 & $\omega \in[300,360]$ & $\mathcal{I}_{\mathrm{MT}}$ & setting 4 \\
\hline
\end{tabular}

Test configuration for Figure 8.5. 

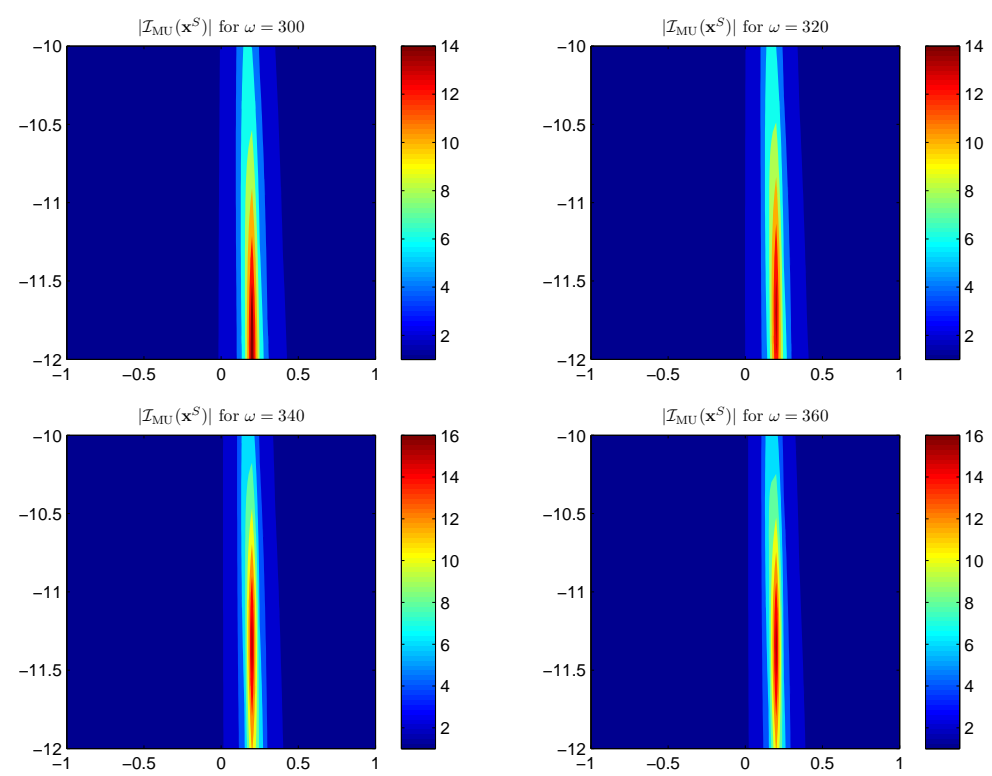

FIG. 8.3. Localization results for $\Sigma_{1}$ using $\mathcal{I}_{M U}$ with $\omega=300,320,340$, and 360 .
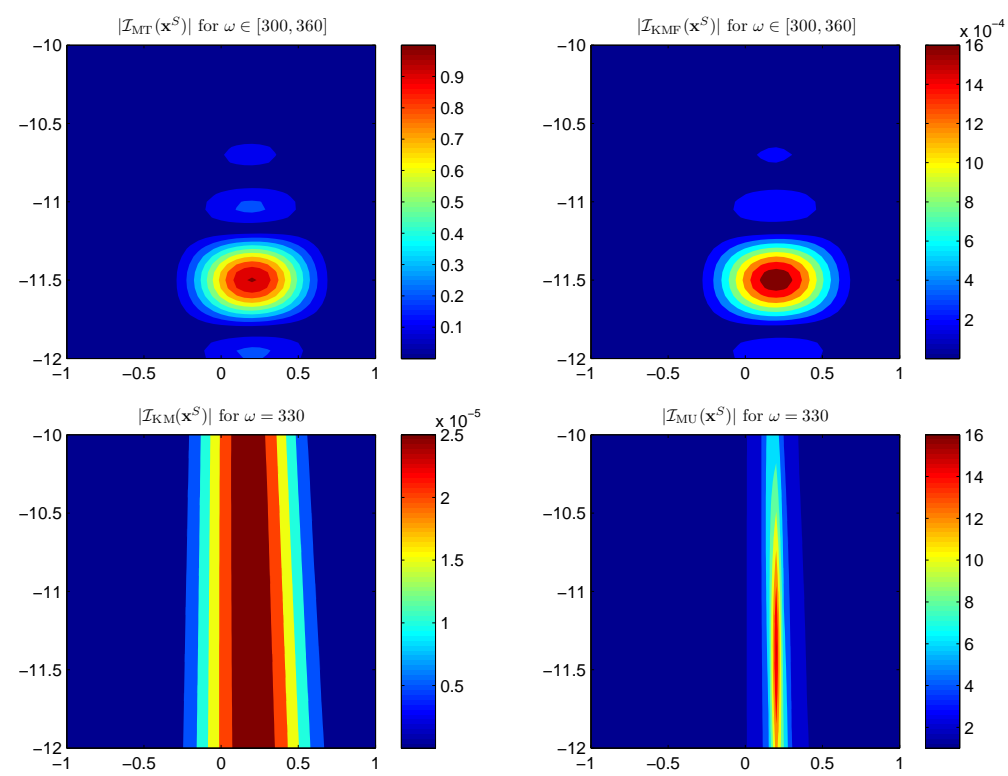

FIG. 8.4. Localization results for $\Sigma_{1}$ using $\mathcal{I}_{\mathrm{MT}}$ (top left), $\mathcal{I}_{\mathrm{KMF}}$ (top right), $\mathcal{I}_{\mathrm{KM}}$ (bottom left) and $\mathcal{I}_{\mathrm{MU}}$ (bottom right). The data set is generated using the asymptotic formula (2.4).

direction $\boldsymbol{t}$ of a single crack minimizes the functional $\mathcal{R}(\boldsymbol{t})$ defined by (7.2). use 8 and 12 search directions $\boldsymbol{t}_{i}$ in the situations with $\Sigma_{1}$ and $\Sigma_{2}$, respectively.

The values of $\mathcal{R}\left(\boldsymbol{t}_{i}\right)$ for the crack $\Sigma_{1}$ are given in Table 8.3. Note that the values of $\mathcal{R}\left(\boldsymbol{t}_{i}\right)$ are normalized with respect to the maximum value.

\begin{tabular}{|c|c|c|c|c|c|c|c|c|}
\hline$l$ & 1 & 2 & 3 & 4 & 5 & 6 & 7 & 8 \\
\hline Value of $\mathcal{R}\left(\boldsymbol{t}_{i}\right)$ & 0.171 & 1.000 & 0.204 & $\mathbf{0 . 0 2 5}$ & 0.171 & 1.000 & 0.204 & $\mathbf{0 . 0 2 5}$ \\
\hline
\end{tabular}

Recovering the direction of the small crack $\Sigma_{1}$. 

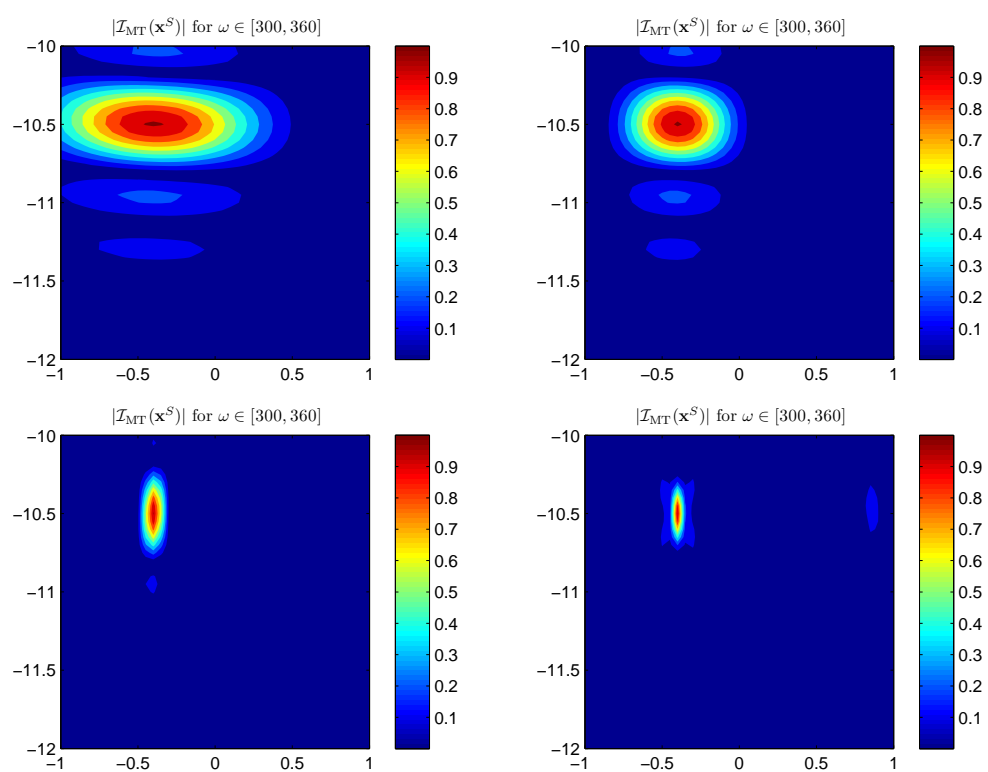

FIG. 8.5. Results for $\Sigma_{2}$ and different arrays. Top left: setting 1, top right: setting 2, bottom left: setting 3 and bottom right: setting 4 .

\begin{tabular}{|c|c|c|c|c|c|c|}
\hline Crack & $n$ & $x_{1}^{(1)}$ & $x_{1}^{(n)}$ & Frequency & Imaging functional & Figure \\
\hline$\Sigma^{(m)}$ & 30 & -1 & 1 & $\omega \in[300,360]$ & $\mathcal{I}_{\mathrm{MT}}, \mathcal{I}_{\mathrm{KMF}}$ & Figs. 8.6, 8.7 \\
\hline$\Sigma^{(m)}$ & 30 & -1 & 1 & $\omega=330$ & $\mathcal{I}_{\mathrm{KM}}, \mathcal{I}_{\mathrm{MU}}$ & Figs. 8.6, 8.7 \\
\hline
\end{tabular}

Test configuration for $\Sigma^{(m)}$.

From the data in Table 8.3, the estimated tangential direction $\hat{\boldsymbol{t}}$ of the crack $\Sigma_{1}$ is given by $\hat{\boldsymbol{t}} \simeq \boldsymbol{t}_{4}, \boldsymbol{t}_{8}=(1,0),(-1,0)$. Thus, the approach set forth for estimation of crack orientation worked well in this case.

8.2. Imaging of Multiple Small Cracks. Consider the functionals $\mathcal{I}_{\mathrm{MT}}$, $\mathcal{I}_{\mathrm{KMF}}, \mathcal{I}_{\mathrm{KM}}$, and $\mathcal{I}_{\mathrm{MU}}$ for imaging multiple cracks. We need to use slight generalizations of formulas (3.10) and (3.5) for $\mathcal{I}_{\mathrm{MT}}$ and $\mathcal{I}_{\mathrm{MU}}$ in which we keep as many singular values as there are cracks. We choose for illustration the following example with two cracks:

$$
\Sigma^{(m)}=\left\{\boldsymbol{x}_{c, 3}+x \boldsymbol{t}_{c, 3}:-l_{c} \leq x \leq l_{c}\right\} \cup\left\{\boldsymbol{x}_{c, 4}+x \boldsymbol{t}_{c, 4}:-l_{c} \leq x \leq l_{c}\right\}
$$

with $\boldsymbol{x}_{c, 3}=(-0.7,-10.8), \boldsymbol{t}_{c, 3}=(1,0), \boldsymbol{x}_{c, 4}=(0.6,-11.3)$, and $\boldsymbol{t}_{c, 4}=(\cos (\pi / 4), \sin (\pi / 4))$.

The test configurations and localization results can be found in Table 8.4 and Fig. 8.6. Note that for $\mathcal{I}_{\mathrm{KMF}}, \mathcal{I}_{\mathrm{KM}}$, and $\mathcal{I}_{\mathrm{MU}}$ there is a peak of much smaller magnitude at the rotated crack (the one on the right). Note also that the normalization of the modes in the functional $\mathcal{I}_{\mathrm{MT}}$ gives a more balanced contrast.

8.3. Robustness With Respect to Measurement Noise. Suppose that the measured data is polluted by a white Gaussian noise so that the SNR is $10 \mathrm{~dB}$. In order to test the robustness of the proposed imaging functionals, we consider the imaging of the two-closely located cracks $\Sigma^{(m)}$. The test configuration is the same as in the noiseless case.

Again, in the imaging of $\Sigma^{(m)}$ a peak of much smaller magnitude at the rotated crack (the one on the right) results when using functionals $\mathcal{I}_{\mathrm{KMF}}$ and $\mathcal{I}_{\mathrm{KM}}$ as shown 

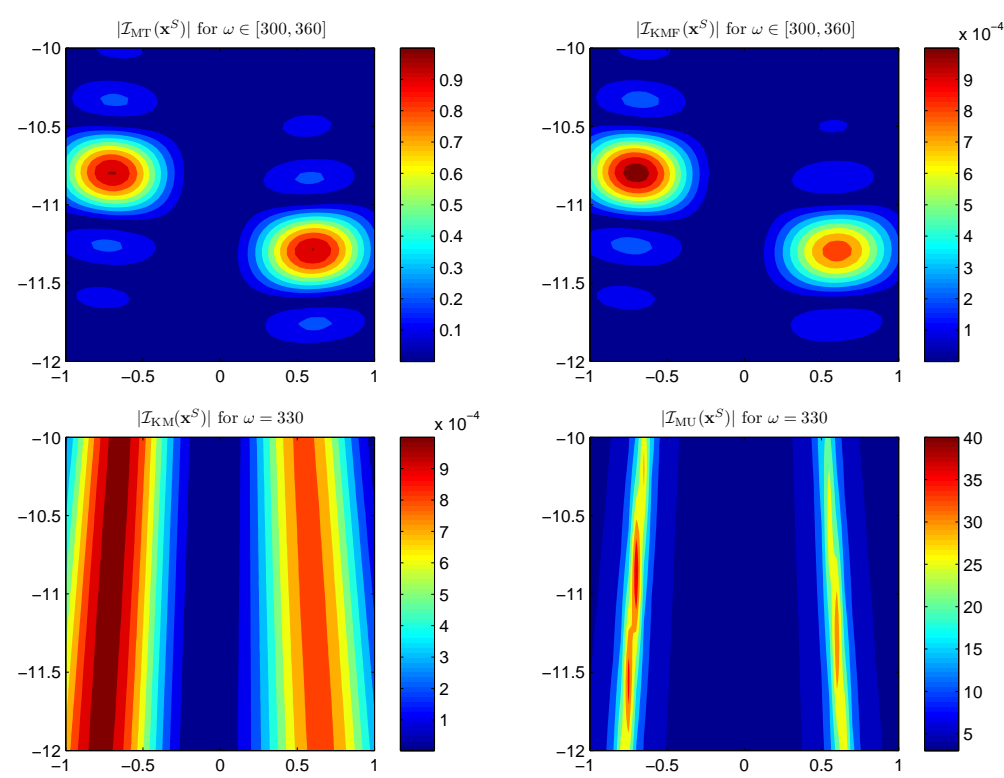

FIG. 8.6. Localization results for $\Sigma^{(m)}$ with $\mathcal{I}_{\mathrm{MT}}$ (top left), $\mathcal{I}_{\mathrm{KMF}}$ (top right), $\mathcal{I}_{\mathrm{KM}}$ (bottom left) and $\mathcal{I}_{\mathrm{MU}}$ (bottom right).
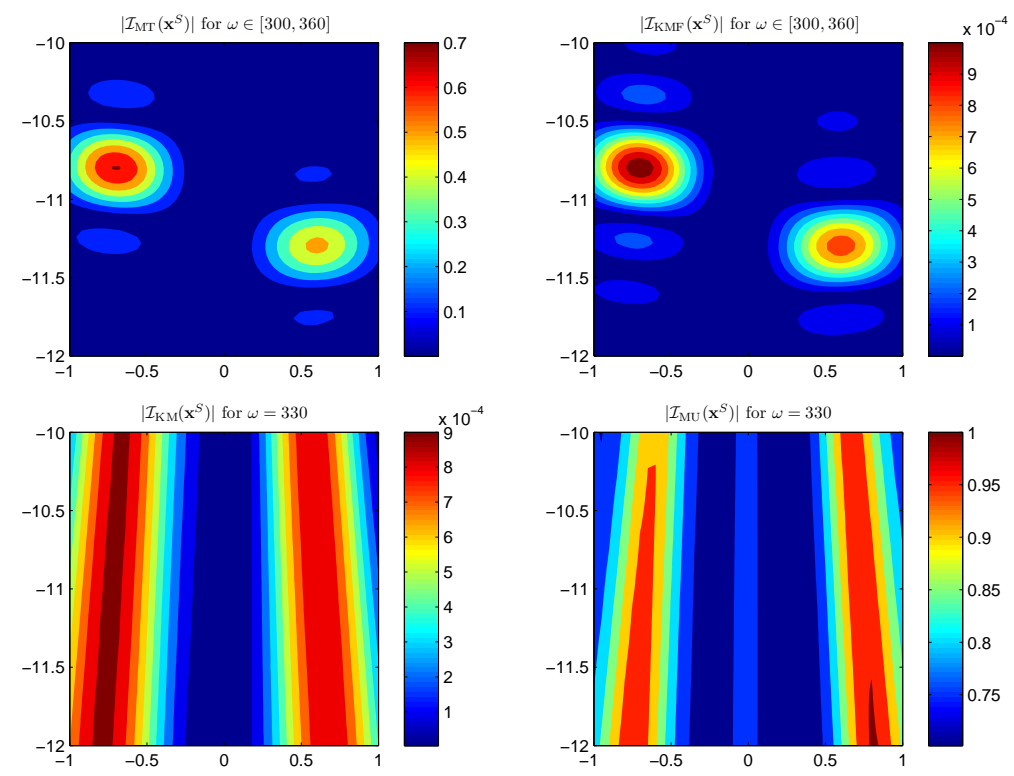

FIG. 8.7. Localization results for $\Sigma^{(m)}$ using $\mathcal{I}_{\mathrm{MT}}$ (top left), $\mathcal{I}_{\mathrm{KMF}}$ (top right), $\mathcal{I}_{\mathrm{KM}}$ (bottom left) and $\mathcal{I}_{\mathrm{MU}}$ (bottom right) with noise with $10 \mathrm{~dB} S N R$.

in Fig. 8.7. Note that KM, KMF, and MT functionals are not significantly affected by measurement noise while $\mathcal{I}_{\mathrm{MU}}$ is much more sensitive to noise.

8.4. Probability of Detection. In this subsection we show the validity of formula (5.1). We fix $\alpha$ and $n=20$ and vary the ratio $\sigma_{0} / \sigma_{\mathrm{c}}$. For each value $\sigma_{0} / \sigma_{\mathrm{c}}$, we generate $1000 \mathrm{MSR}$ matrix, by adding a Gaussian noise to a MSR matrix corresponding to a small crack. Then, we compute the ratio $R$ for each noisy matrix and then count the number of matrices such that $R$ is larger than $r_{\alpha}$.

The theoretical curve for the probability of detection given by (5.1) and the 

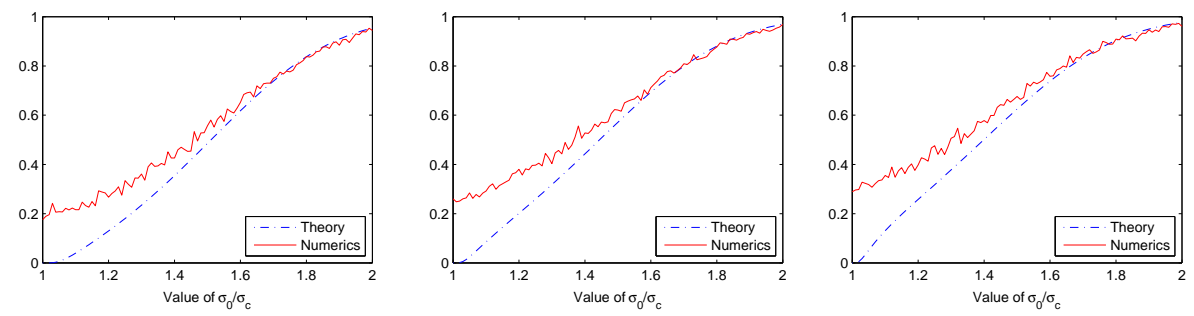

Fig. 8.8. Comparison between simulated and theoretical PODs for $\alpha=0.01$ (left), 0.05 (middle), and 0.1 (right), respectively.

simulated ones as a function of the ratio $\sigma_{0} / \sigma_{\mathrm{c}}$ are compared in Fig. 8.8. We can see that the higher the ratio $\sigma_{0} / \sigma_{\mathrm{c}}$, the better the accuracy of (5.1) is.

9. Conclusion. We have presented a framework for detection and localization of cracks. The approach is based on physical modelling, modelling the formation of the response function using the governing equation for the wave propagation phenomenon and evaluating this via asymptotic techniques. The explicit tests are then based on singular value decomposition statistical decision rules in the context when there is additive Gaussian noise. An important aspect of the detection rule is that it is universal in that it depends only on the number of measurements and the prescribed false alarm rate. The approach also addresses the important question of identifying the dimension of the signal space. Our probabilistic approach identifies a Kirchhoff imaging functional as the optimal one in the localization step. Finally, the asymptotic framework answers the question about what aspects of cracks can be estimated based on the measurements and we set explicit schemes of estimation of these geometric features.

Our approach in this paper can be extended for imaging perfectly insulating small cracks (i.e., with Neumann boundary conditions). The approximation is in this case a dipole-type one. The framework that we have presented can also be generalized to the case with cluttered media and the situation with multiple and clustered defaults. This requires to determine the statistical properties of the MSR matrix in this framework, using the theory of wave propagation in complex media [19]. Results on this will be reported elsewhere.

Appendix A. Statistical Test. As in the standard statistical hypothesis testing $[16,21]$, we postulate two hypotheses and derive a decision rule for deciding in between them based on the measured MSR matrix.

We define $H_{0}$ the (null) hypothesis to be tested and $H_{A}$ the (alternative) hypothesis:

- $H_{0}$ : there is no crack,

- $H_{A}$ : there is a crack.

We want to test $H_{0}$ against $H_{A}$. Two types of independent errors can be made:

- Type I errors correspond to rejecting the null hypothesis $H_{0}$ when it is correct (false alarm). Their probability is given by

$$
\alpha:=P\left[\text { accept } H_{A} \mid H_{0} \text { true }\right] .
$$

- Type II errors correspond to accepting $H_{0}$ when it is false (missed detection) and have probability

$$
\beta:=P\left[\text { accept } H_{0} \mid H_{A} \text { true }\right] .
$$

The success of the test (probability of detection or detection power) is therefore given by $1-\beta$. 
Given the data the decision rule for accepting $H_{0}$ or not can be derived from the Neyman-Pearson lemma which asserts that for a prescribed false alarm rate $\alpha$ the most powerful test corresponds to accepting $H_{A}$ for the likelihood ratio of $H_{A}$ to $H_{0}$ exceeding a threshold value determined by $\alpha$.

Neyman-Pearson Lemma: Let $Y$ be the set of all possible data and let $f_{0}(y)$ and $f_{1}(y)$ be the probability densities of $Y$ under the null and alternative hypotheses. The Neyman-Pearson lemma [16, p. 335] states that the most powerful test has a critical region defined by

$$
\mathcal{Y}_{\alpha}:=\left\{y \in Y \mid \frac{f_{1}(y)}{f_{0}(y)} \geq \eta_{\alpha}\right\},
$$

for a threshold $\eta_{\alpha}$ satisfying

$$
\int_{y \in \mathcal{Y}_{\alpha}} f_{0}(y) d y=\alpha
$$

If the data is $y$, we reject $H_{0}$ if the likelihood ratio $\frac{f_{1}(y)}{f_{0}(y)}>\eta_{\alpha}$ and accept $H_{0}$ otherwise. The power of the (most powerful) test is

$$
1-\beta=\int_{y \in \mathcal{Y}_{\alpha}} f_{1}(y) d y
$$

Appendix B. Proof of the Uniform Asymptotic Formula (2.4). Assume for the sake of simplicity that $\boldsymbol{x}_{c}=\mathbf{0}$ and $\boldsymbol{t}=(1,0)$ so that

$$
\Sigma_{c}=\left\{(x, 0):-l_{c} \leq x \leq l_{c}\right\} .
$$

We will need the two following lemmas in this appendix.

Lemma B.1. Cf. [5, Lemma 2.1]. The weighted $L^{2}$-space $\mathcal{X}$ defined by

$$
\mathcal{X}=\left\{\varphi: \int_{-1}^{1} \sqrt{1-x^{2}}|\varphi(x)|^{2} d x<+\infty\right\}
$$

is a Hilbert space. The operator $\mathcal{L}$ defined by

$$
\mathcal{L} \varphi(x)=\int_{-1}^{1} \varphi(y) \ln |x-y| d y
$$

is invertible from $\mathcal{X}$ to $\mathcal{Y}=\left\{\varphi \in \mathcal{C}^{0}(-1,1), \varphi^{\prime} \in \mathcal{X}\right\}$ and its inverse is

$$
\mathcal{L}^{-1} \varphi(x)=-\frac{1}{\pi^{2} \sqrt{1-x^{2}}} \int_{-1}^{1} \frac{\sqrt{1-y^{2}} \varphi^{\prime}(y)}{x-y} d y+\frac{a(\varphi)}{\pi \ln (1 / 2) \sqrt{1-x^{2}}}
$$

where $a(\varphi)$ is a constant.

Lemma B.2. Cf. [1, Chap. 9]. The following behavior of $H_{0}^{(1)}$ near 0 holds:

$$
-\frac{i}{4} H_{0}^{(1)}(r)=\frac{1}{2 \pi} \ln r+\alpha+\sum_{n=1}^{+\infty}\left(\beta_{n} \ln r+\theta_{n}\right) r^{2 n}, \text { for } r \ll 1,
$$

where

$$
\beta_{n}=\frac{(-1)^{n}}{2 \pi} \frac{1}{2^{2 n}(n !)^{2}}, \quad \theta_{n}=-\beta_{n}\left(\gamma-\ln 2-\frac{\pi i}{2}-\sum_{j=1}^{n} \frac{1}{j}\right)
$$


and the constant $\alpha=(\gamma-\ln 2) /(2 \pi)-i / 4, \gamma$ being the Euler constant.

The following behavior of $H_{0}^{(1)}$ and its derivative at infinity holds:

$\frac{i}{4} H_{0}^{(1)}(r) \simeq \frac{1}{\sqrt{8 \pi r}} \exp \left(i r+i \frac{\pi}{4}\right), \quad \frac{i}{4} \frac{d}{d r} H_{0}^{(1)}(r) \simeq \frac{1}{\sqrt{8 \pi r}} \exp \left(i r+i \frac{3 \pi}{4}\right), \quad$ for $r \gg 1$.

We have the integral representation of the field $u^{(j)}$ emitted by a source at $\boldsymbol{x}_{j}$ for $\boldsymbol{x} \neq \boldsymbol{x}_{j}$ :

$$
u^{(j)}(\boldsymbol{x})=\hat{G}\left(\omega, \boldsymbol{x}, \boldsymbol{x}_{j}\right)+\int_{-l_{c}}^{l_{c}} \phi^{(j)}(y) \hat{G}(\omega,(y, 0), \boldsymbol{x}) d y
$$

where the density $\phi^{(j)}$ is the solution to the integral equation

$$
\int_{-l_{c}}^{l_{c}} \phi^{(j)}(y) \hat{G}(\omega,(y, 0), \boldsymbol{x}) d y=-\hat{G}\left(\omega, \boldsymbol{x}, \boldsymbol{x}_{j}\right), \quad \text { for } \boldsymbol{x}=(x, 0) \in \Sigma_{c} .
$$

Using (2.2) the normalized function $\tilde{\phi}^{(j)}(\tilde{y})=l_{c} \phi^{(j)}\left(l_{c} \tilde{y}\right)$ is solution to

$$
\int_{-1}^{1} \tilde{\phi}^{(j)}(\tilde{y})\left[-\frac{i}{4} H_{0}^{(1)}\left(\varepsilon_{\omega}|\tilde{x}-\tilde{y}|\right)\right] d \tilde{y}=\frac{i}{4} H_{0}^{(1)}\left(\left|\frac{\omega}{c_{0}} \boldsymbol{x}_{j}-\varepsilon_{\omega}(\tilde{x}, 0)\right|\right) \quad \text { for } \tilde{x} \in(-1,1) .
$$

In the regime $\varepsilon_{\omega} \ll 1$, for any $\tilde{x} \in(-1,1)$ the right-hand side can be expanded as

$$
\frac{i}{4} H_{0}^{(1)}\left(\left|\frac{\omega}{c_{0}} \boldsymbol{x}_{j}-\varepsilon_{\omega}(\tilde{x}, 0)\right|\right)=\frac{i}{4} H_{0}^{(1)}\left(\left|\frac{\omega}{c_{0}} \boldsymbol{x}_{j}\right|\right)-\varepsilon_{\omega} \tilde{x} \frac{\partial}{\partial x} \frac{i}{4} H_{0}^{(1)}\left(\left|\frac{\omega}{c_{0}} \boldsymbol{x}_{j}\right|\right)+O\left(\varepsilon_{\omega}^{2}\right),
$$

and we get from (B.3) that the left-hand side can be expanded as

$\int_{-1}^{1} \tilde{\phi}^{(j)}(\tilde{y})\left[-\frac{i}{4} H_{0}^{(1)}\left(\varepsilon_{\omega}|\tilde{x}-\tilde{y}|\right)\right] d \tilde{y}=\frac{1}{2 \pi} \mathcal{L} \tilde{\phi}^{(j)}(\tilde{x})+\left(\frac{1}{2 \pi} \ln \varepsilon_{\omega}+\alpha\right) C^{(j)}+O\left(\varepsilon_{\omega}^{2} \ln \varepsilon_{\omega}\right)$,

where $O\left(\varepsilon_{\omega}^{2} \ln \varepsilon_{\omega}\right)$ is in the $\mathcal{X}$-norm and

$$
C^{(j)}=\int_{-1}^{1} \tilde{\phi}^{(j)}(\tilde{y}) d \tilde{y}
$$

It then follows that $\tilde{\phi}^{(j)}$ is the solution in $\mathcal{X}$ to

$\frac{1}{2 \pi} \mathcal{L} \tilde{\phi}^{(j)}(\tilde{x})=-C^{(j)}\left(\frac{\ln \varepsilon_{\omega}}{2 \pi}+\alpha\right)+\hat{G}\left(\omega, \boldsymbol{x}_{c}, \boldsymbol{x}_{j}\right)+\varepsilon_{\omega} \tilde{x} \frac{\partial \hat{G}}{\partial x}\left(\omega, \boldsymbol{x}_{c}, \boldsymbol{x}_{j}\right)+O\left(\varepsilon_{\omega}^{2} \ln \varepsilon_{\omega}\right)$

where $\boldsymbol{x}_{c}$ is the location of the crack and $\partial / \partial x$ is the tangential derivative on the crack $\Sigma_{c}$ defined by (B.1). Using the explicit form of $\mathcal{L}^{-1}$ yields

$$
\begin{array}{r}
\tilde{\phi}^{(j)}(\tilde{x})=C^{(j)} \frac{\ln \varepsilon_{\omega}+2 \pi \alpha}{\pi \ln (2) \sqrt{1-\tilde{x}^{2}}}-\frac{2 \hat{G}\left(\omega, \boldsymbol{x}_{c}, \boldsymbol{x}_{j}\right)}{\ln (2) \sqrt{1-\tilde{x}^{2}}}-2 \varepsilon_{\omega} \frac{\partial \hat{G}}{\partial x}\left(\omega, \boldsymbol{x}_{c}, \boldsymbol{x}_{j}\right) \frac{\tilde{x}}{\sqrt{1-\tilde{x}^{2}}} \\
+O\left(\varepsilon_{\omega}^{2} \ln \varepsilon_{\omega}\right),
\end{array}
$$

and therefore (by integrating in $\tilde{x}$ )

$$
C^{(j)}=\frac{2 \pi \hat{G}\left(\omega, \boldsymbol{x}_{c}, \boldsymbol{x}_{j}\right)}{\ln \left(\varepsilon_{\omega} / 2\right)+2 \pi \alpha}+O\left(\varepsilon_{\omega}^{2} \ln \varepsilon_{\omega}\right) .
$$


Finally, plugging the expansion of $\tilde{\phi}^{(j)}$ in (B.5) we get the asymptotic formula for $A_{j l}=u^{(j)}\left(\boldsymbol{x}_{l}\right)$ :

$$
A_{j l}=\frac{2 \pi \hat{G}\left(\omega, \boldsymbol{x}_{j}, \boldsymbol{x}_{c}\right) \hat{G}\left(\omega, \boldsymbol{x}_{c}, \boldsymbol{x}_{l}\right)}{\ln \left(\frac{\varepsilon_{\omega}}{4}\right)+\gamma-\frac{i \pi}{2}}-\pi l_{c}^{2} \frac{\partial}{\partial x} \hat{G}\left(\omega, \boldsymbol{x}_{j}, \boldsymbol{x}_{c}\right) \frac{\partial}{\partial x} \hat{G}\left(\omega, \boldsymbol{x}_{c}, \boldsymbol{x}_{l}\right)+\mathrm{o}\left(\varepsilon_{\omega}^{2}\right),
$$

which is uniform in $\varepsilon_{\omega}$. If, additionally, we have $\omega L / c_{0} \gg 1$, then the Green's function can be expanded using the asymptotic form (B.4) of $H_{0}^{(1)}$ and we get finally the form (2.4).

\section{REFERENCES}

[1] M. Abramowitz and I. Stegun, Handbook of mathematical functions, Dover Publications, New York, 1965.

[2] R. Adamczak, A few remarks on the operator norm of random Toeplitz matrices, J. Theor. Probab., DOI 10.1007/s10959-008-0201-7 (2009).

[3] N. I. Akhiezer and I. M. Glazman, Theory of Linear Operators on Hilbert Spaces, Vol. 1, Dover, New York, 1993.

[4] H. Ammari, H. Kang, and H. Lee, Layer Potential Techniques in Spectral Analysis, Mathematical Surveys and Monographs, Vol. 153, American Mathematical Society, Providence RI, 2009.

[5] H. Ammari, H. Kang, H. Lee, and W.K. Park, Asymptotic imaging of perfectly conducting cracks, SIAM J. Sci. Comput. 32, 894-922 (2010).

[6] G. Bal and O. Pinaud, Time reversal based detection in random media, Inverse Problems 21, 1593-1620 (2005).

[7] A. P. Berens, NDE reliability data analysis, ASM Handbook, Vol. 17, 689-701 (1989).

[8] L. Borcea, G. Papanicolaou, and C. Tsogka, Adaptive interferometric imaging in clutter and optimal illumination, Inverse Problems 22, 1405-1436 (2006).

[9] A. Bose and A. Sen, Spectral norm of random large dimensional noncentral Toeplitz and Hankel matrices, Electron. Comm. Probab. 12, 29-35 (2007).

[10] M. Brühl, M. Hanke, and M. Pidcock, Crack detection using electrostatic measurements, Math. Model. Numer. Anal. 35, 595-605 (2001).

[11] K. Bryan and M. S. Vogelius, A review of selected works on crack identification, in Geometric Methods in Inverse Problems and PDE Control, IMA Vol. 137, Springer-Verlag, Berlin, 2004.

[12] W. Bryc, A. Dembo, and T. Jiang, Spectral measure of large random Hankel, Markov and Toeplitz matrices, Ann. Probab. 34, 1-38 (2006).

[13] M. Capitaine, C. Donati-Martin, and D. Féral, The largest eigenvalue of finite rank deformation of large Wigner matrices: convergence and nonuniversality of the fluctuations, Ann. Probab. 37, 1-47 (2009).

[14] G. Casella and R. L. Berger, Statistical Inference, Duxbury Press, Pacific Grove, 2002.

[15] S. Chatterjee, Fluctuations of eigenvalues and second order Poincaré inequalities, Probab. Theory Relat. Fields 143, 1-40 (2009).

[16] A. C. Davison, Statistical Models, Cambridge University Press, Cambridge, 2003.

[17] A. R. Elcrat, V. Isakov, and O. Neculoiu, On finding a surface crack from boundary measurements, Inverse Problems 11, 343-351 (1995).

[18] D. Féral and S. Péché, The largest eigenvalue of rank one deformation of large Wigner matrices, Comm. Math. Phys. 272, 185-228 (2006).

[19] J.-P. Fouque, J. Garnier, G. Papanicolaou, and K. Sølna, Wave Propagation and Time Reversal in Randomly Layered Media, Springer, New York, 2007.

[20] I. M. Johnstone, On the distribution of the largest eigenvalue in principal component analysis, The Annals of Statistics 29, 295-327 (2001).

[21] S. M. Kay, Fundamentals of Statistical Signal Processing, Detection Theory, Englewood Cliffs, Prentice-Hall, 1998.

[22] M. Meckes, On the spectral norm of a random Toeplitz matrix, Elect. Comm. in Probab. 12 , 315-325 (2007).

[23] M. L. Mehta, Random Matrices, Academic Press, San Diego, 1991.

[24] G. A. F. Seber and C. J. Wild, Nonlinear Regression, Wiley, Hoboken, 2003. 